\title{
The use of robotics devices in knee rehabilitation: a critical review
}

\author{
R. Wilmart ${ }^{1}$, E. Garone ${ }^{1}$, B. Innocenti ${ }^{2}$ \\ 1 SAAS Department, Université Libre de Bruxelles, Bruxelles, Belgium \\ 2 BEAMS Department, Université Libre de Bruxelles, Bruxelles, Belgium
}

\author{
CORRESPONDING AUTHOR \\ Bernardo Innocenti \\ BEAMS Department \\ (Bio Electro and Mechanical \\ Systems), \\ Université Libre de Bruxelles \\ Av. F. Roosevelt 50 \\ 1050 Bruxelles, Belgium \\ E-mail: \\ bernardo.innocenti@ulb.ac.be
}

DOI

10.32098/mltj.01.2019.07

\begin{abstract}
SUMMARY
Knee surgery is a common procedure to treat cartilage defects, soft tissue lesions as cruciate ligaments (ACL/PCL), and osteoarthritis with total or unicondylar knee arthroplasty. After knee surgery, every patient undergoes a long period of rehabilitation (typically from 6 weeks to 6 months) consisting of long sessions of physiotherapy and medical training therapy carried out by qualified personnel. This procedure is long and expensive, and may cause work-related pathologies to physiotherapists. Fortunately, it is generally agreed that robotics may benefit to both patients and physiotherapists due to its ability to repeat tasks with accuracy and its potential to measure the progress of the rehabilitation. This paper aims at providing a critical review of the different proposed robotic solutions and the associated rehabilitation techniques for the knee in particular and for the lower limb in general, with the sake of highlighting the pros and cons and to identify possible promising directions of research.
\end{abstract}

\section{KEY WORDS}

Biomechanics, Continuous Passive Motion (CPM), Exoskeletons, Gait-trainers, Self-aligning, Therapeutic Exercise Machines

\section{INTRODUCTION}

Knee surgery is a common procedure to treat cartilage defects, soft tissue lesions as cruciate ligaments injuries, and osteoarthritis with total or unicompartmental knee arthroplasty. Among the different surgical procedures, knee replacement and anterior cruciate ligament reconstruction are the most common surgeries. To provide some number, in 2016 the health division of the Organization for Economic Co-operation and Development (OECD) reported that more than 950,000 knee replacement surgeries were performed in Europe in 2013, with 21,208 knee replacement surgeries only in Belgium (1). This means almost 10.2 patients operated every hour in a working day of 8 hours. It has also been estimated that there are 200,000 new ACL injuries every year only in the United States, half of which occurring along with damage to other structures of the knee and requiring a surgical intervention (2). In 2012 around 4500 primary and revision ACL surgeries were performed in Belgium (annual incidence around 40/100,000 inhabitants) (3).

After knee surgery, every patient undergoes a long period of rehabilitation (typically from 6 weeks to 6 months) consisting of long sessions of physiotherapy and medical training therapy carried out by qualified personnel (4). This procedure is long and expensive, and may cause work-related pathologies to physiotherapists due to the significant workload it requires (5). These considerations highlight the high potential benefits that robotic solutions can bring in this field. As a matter of fact, if technology could provide an effective tool to assist the physiotherapist, the rehabilitation time and cost would be reduced, with important benefits for both the patients and the operators.

The first research activities in this direction date back to 1975 when Dr. Robert B. Salter et al. (6) proposed the first continuous passive motion (CPM) machine, which can be considered as the first robotic machine designed for knee rehabilitation. Since then, several knee robotic rehabilitation devices have been introduced in the scientific literature and proposed on the market.

This study aims at providing a critical review of the different proposed robotic solutions and the associated rehabilitation techniques for lower limb, focusing mainly on the knee joint, with the aim of highlighting the pros and cons and to identify possible promising directions of research and development. The paper is organized as follows. In the section "Rehabilitation Robotics" we will first survey the existing robotic machines and detail for each of them the application field, 
the design choices, the control strategies and, when available, the results of the clinical trials.

In the section "Discussion", we will discuss the pros and cons of existing lower limbs rehabilitation devices. Finally, in the section "Conclusion" we will draw some conclusions and we will highlight possible research directions to develop better lower limb rehabilitation devices.

\section{REHABILITATION ROBOTICS}

A good definition of rehabilitation robotics is the one given by Eduardo Rocon et al. (7): "Rebabilitation robotics has been defined as the combination of industrial robotics and medical rehabilitation, thus encompassing many areas, including mechanical and electrical engineering, biomedical engineering, artificial intelligence, and sensor and actuator technology. Medical rehabilitation often refers to the process by which human function, be it physical or cognitive, is restored at least partially to their "normal" condition. Strictly speaking, Rebabilitation Robotics would not encompass systems that aim at replacing the mechanical function of weak or missing human limbs. However, in an integrative view, here we also consider functional restoration as an important area within Rebabilitation Robotics".

Several different classifications of rehabilitation robots have been proposed in the literature. Classically, the field of rehabilitation robotics is divided into two categories: therapy or assistance robots; depending if they are used with the aim of recovery from an injury (the first), or for assistance of impaired limbs in daily life activities (the second) (8).

Another standard classification is to distinguish if the robots are fixed-based systems or wearable robots (7). Fixed-based systems are, as their name suggests, fixed structures with a stationary coordinate system with respect to the floor (see e.g. figure 1). A special case of fixed-based systems are

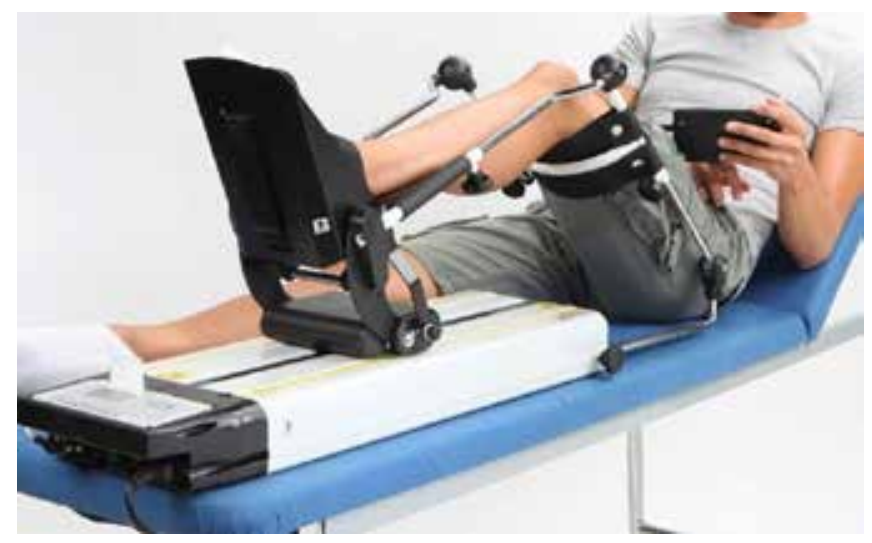

Figure 1 robotic arms that guide the patient's limb along predefined trajectories. They are called "end-effector systems" because the interaction between the driven member and the robotic arm often occurs at the end of the kinematic chain. When they are used for lower extremities, they consist of devices that guide the foot motion without constraining the multiple degrees of freedom of the rest of the body (e.g. knee, hip). The other main class of fixed-based systems are robots whose components are attached to several parts of the patient body and actuated at different joints thus controlling and/or constraining multiple degrees of freedom of the kinematics of the leg. Examples of this class of fixed-based systems are most of the gait training machines.

Wearable robots are robotics systems that a person wears to enhance his/her capabilities. Contrary to fixed-based systems, their coordinate systems are attached to the human body and they constantly move with respect to the ground (10). They are person-oriented robots and can be worn by human operators, whether to enhancement the function of a limb or to replace it completely (11). A wearable robot is designed to match the shape and function of the human body. Their original application was power amplification (e.g. the powered exo-skeleton "HardiMan" (figure 2) 1965 or Kazerooni's extender, 1990). Later they have been proposed for applications such as rehabilitation and assistive devices for disabled or elderly people (7).

According on whether they substitute missing limbs (for instance due to an amputation) or if they operate alongside human limbs to treat or assist a patient, they are called either prosthesis or orthotic robots/exoskeletons.

In this paper we use a slightly different classification of rehabilitation robots. In particular, we classify the rehabilitation robots according to their operative mode contrasting with the classical classification based on their kinematics. Consequently, we consider the following three categories:

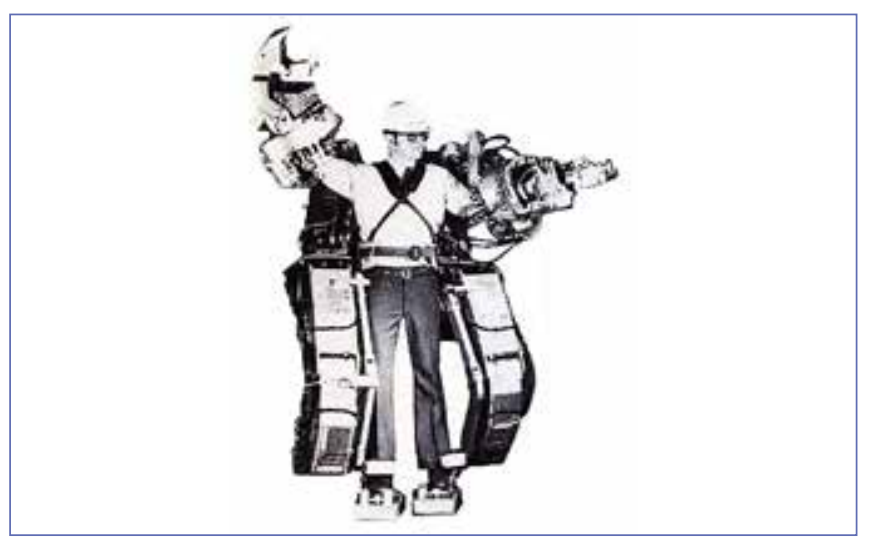

Figure 2 
- Continuous Passive Motion (CPM) machines and Therapeutic Exercise Machines (TEM);

- Gait-training Robots;

- Exoskeletons.

Hereafter, we will analyze these three groups of robots for the rehabilitation of lower limb (and of the knee in particular) trying to put in evidence the advantages and disadvantages of each presented robot, and, when available, surveying the results of the associated medical trials.

\section{Continuous Passive Motion (CPM) Machines and Therapeutic Exercise Machines}

The concept of CPM was introduced in 1970 by Robert B. Salter (13) for rehabilitation after reconstructive joint surgery such as knee replacement and ACL reconstruction. In 1978, the first CMP device was developed in collaboration with the engineer John Saringer (13). Since then, the use of CPM machines (e.g. the FISIOTEK 3000 TSF, figure 3 a) has spread in rehabilitation centers and hospitals for post-knee surgery treatments and is currently the most used rehabilitation device for the lower limbs (14).

CPM devices are a family of fixed-base end-effector systems which are guided by one motor with a variable range of velocity and movement. Usually, they are not programmable and they are controlled in open loop without any force or position feedback. From the kinematic viewpoint, a CPM can be described as a two bars system (see figure $3 \mathbf{b}$ ), operating in a 2D plan and articulated at the hip, knee and ankle, each considered as a hinge joint. The force is generated by the robot on the patient's foot. As a consequence, a CPM can only provide movement in one plane, usually the sagittal plane (10). CPM devices constantly move the knee joint through a pre-defined range of motion. Throughout the rehabilitation process this range of motion is usually increased over time, with the goal of increasing the joint mobility. One of the main limitations of CPMs is that, as its name suggests, they are passive machines, i.e. they do not require the user to exert any force during the motion. As a consequence, the patient does not actively move his leg using a CPM machine. Moreover, the device only moves the knee in flexion/extension.

Several clinical studies have been carried out to evaluate the effectiveness of the use of CPM devices after total knee arthroplasty (TKA) and/or anterior cruciate ligament (ACL) reconstruction. However, the results are quite controversial $(15,16)$. On the one hand, some studies conclude that CPM has no benefit for patients following ACL reconstructions or in immediate functional recovery post-TKA $(11,12)$. These studies also claim that CPM machines do not provide any additional benefits on knee extension Range of Motion (ROM), functional ability, or Length of Stay (LOS), and that they can even increase the postoperative knee swelling time and the required doses of analgesics (17). Furthermore, during these trials it was observed that, during the rehabilitation process, patients might move their extremities suddenly due to reflexes. As CPM machines cannot handle such behavior, the reflex of a patient can move his leg while the machine is operating and result in an improper load in the joint that can damage the patient's muscles or tendons (18). On the other hand, others studies claim that CPM devices are very effective in preserving the range of movement, reduce stiffness in joints, decrease the need for drug administration, and shorten the length of hospitalization $(10,19,20)$. Kent Boese et al. (21) suggests that this controversy might be due to an unavoidable bias in any CPM study: in most of the clinical trials, the patient was free to stop the CPM machines whenever he wanted, to ensure subjects' safety and comfort. As a consequence, there is a bias on the trial duration when comparing it with the traditional rehabilita-

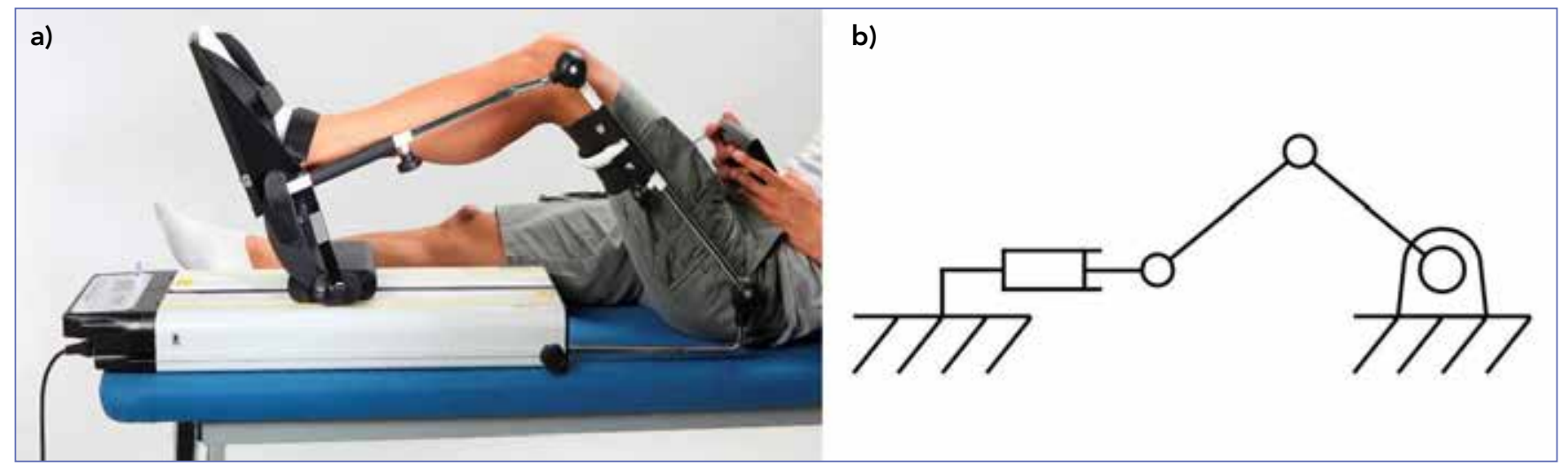

Figure 3 
tion sessions whose duration is not set by the patient but by the physiotherapist.

However, scientific literature seems to agree on the fact that the use of CPM devices might result in weaker muscles, longer delays in activation of extensors and higher stiffness of flexors (10) if compared with traditional physiotherapy. A possible explanation is that, as shown by several recent rehabilitation studies (4), to enhance the effectiveness of the rehabilitation the patient should be actively involved in the rehabilitation process since the very beginning.

Because of these controversies and for the sake of improving the recovery, other machines have been developed aiming at enhancing the activity of the patient during the rehabilitation process.

In 1988, a first step in this direction was the development of BioDex (figure 4). This robot is a programmable force-controlled, single-axis device for muscle exercise and rehabilitation which consists of a movable fixture against which an actuating force can be applied. This machine has been developed to treat muscular lesions and cruciate ligament lesions (22). The same year, the first multi-axis concept was published by Khalili and Zomlefer (23), which resulted in the development of the RTX manipulator (24) (figure 5), an upper-extremity rehabilitation device directly inspired from the multi-axis concept.

In 2001, Moughamir et al. (26) proposed a computer-controlled machine for the training and rehabilitation of the lower limbs called Multi-Iso. Multi-Iso is a fixed-based machine consisting of a chair and a moving part articulated at the knee joint by the use of a single hinged joint. During the exercise sessions, the patient remains seated while his leg is clamped at the ankle and at the thigh. Thanks to six motors, the position of the seat can be adapted, either manually or automatically to a memorized position, so as to suit the requirements and the morphology of each user. Multi-

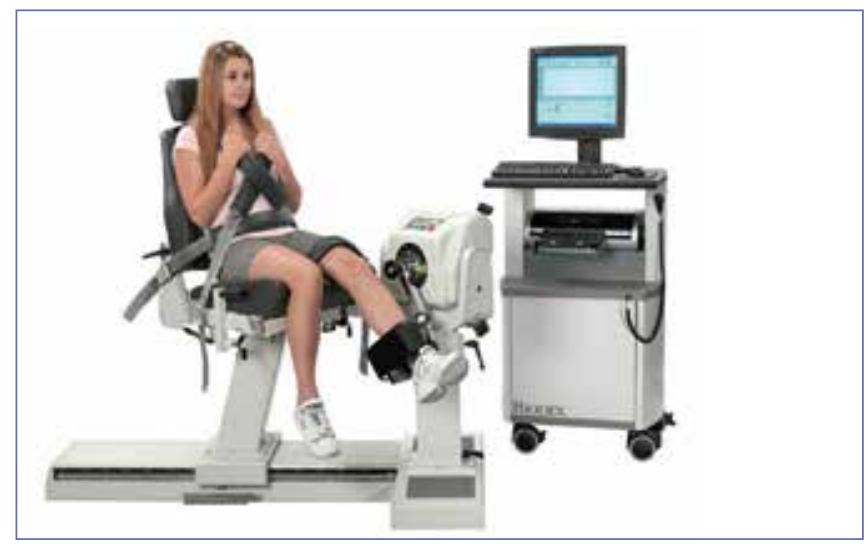

Figure 4
Iso can carry out seven different training modes (Isokinetic, Steering, Isometric, Isotonic, Physiokinetic, Stretching, and Assisted). To perform the required movement patterns for each specific training session, the Multi-Iso could use three different possible parameters (as position, velocity, and force) using different sequences. Such sequences depend on the nature of the exercises, the angular position of the knee in flexion-extension, and the patient's resistive force. The "force" is used to simulate a variable mechanical load whose weight can be chosen through a man-machine interface to reproduce the behavior of a classical weight training machine. The "position" is used when the patient needs to apply a maximal force around a fixed number of positions determined by the physiotherapist. The "velocity" is essentially used during Isokinetic and Steering training or rehabilitation modes to guarantee a constant velocity without static error (26). A campaign of clinical trials has been carried out for the Multi-ISO. However, the clinical trials (26) were made on a group of five healthy sport-women, which does not allow to evaluate its effectiveness for rehabilitation of the lower limbs.

In 2009, a rehabilitation machine called NeXOS (see figure 6) was developed at the University of Abertay Dundee, UK. The developers proposed a device whose main target is stroke rehabilitation. NeXOS is a fixed-based system consisting of three moving parts, two of which are in direct contact with the leg. The third part is a pneumatic linear motor articulated at the foot. Another one is attached to the first moving part and allows it to rotate around a fix point. The machine structure allows NeXOS to move the leg in a range of motion much closer to the one proposed by the physiotherapist. The main feature of this machine is that, in addition to its ability to passively move the leg through a defined series of movements, it allows to carry out a wide set of operations: motion may range from the purely passive,

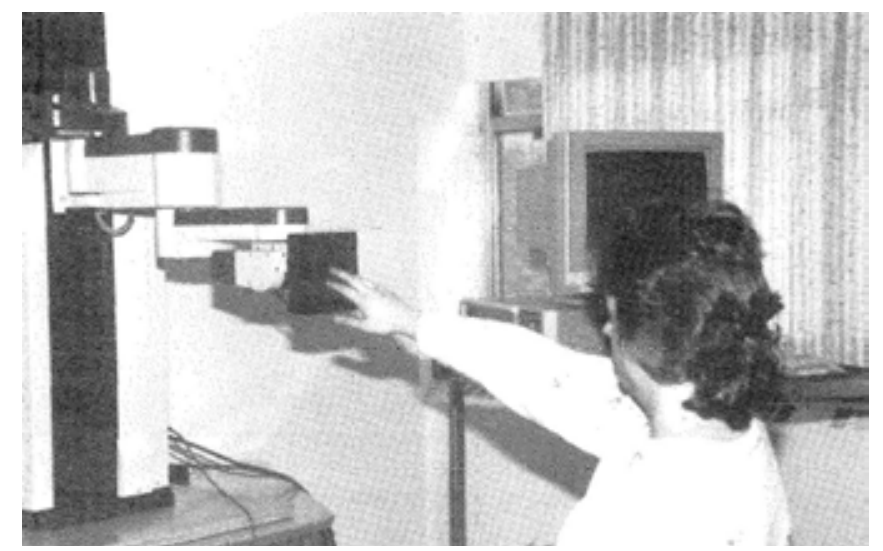

Figure 5 
to assisted and resistive modes, where the patient works against the system. It is important to note that NeXOS is able to assist the user's movements. This is done through a compliant system that does not force the patient to execute a movement but help him to actively achieve his target (27). At the best of our knowledge, no clinical study on this kind of machine is reported in the literature.

Four years later (2011), the Physiotherabot (figure 7) was proposed along the same philosophy of actively involve the patient in the rehabilitation process. Physiotherabot is a 3 DOFs therapeutic exercise robot for the lower limbs of a patient who needs rehabilitation after a spinal cord injury (SCI), stroke, muscle disorder, or surgical operation. It can perform both active and passive exercises. Physiotherabot is programmable, i.e. it can learn specific exercise motions and perform them on a patient without the help of a physiotherapist. It is also important to remark that, similarly to NeXOS, Physiotherabot is a compliant robot, i.e. if the patient reacts against the robot manipulator during the exercise, Physiotherabot will react in such a way to not hurt the patient. Physiotherabot can perform flexion-extension movement

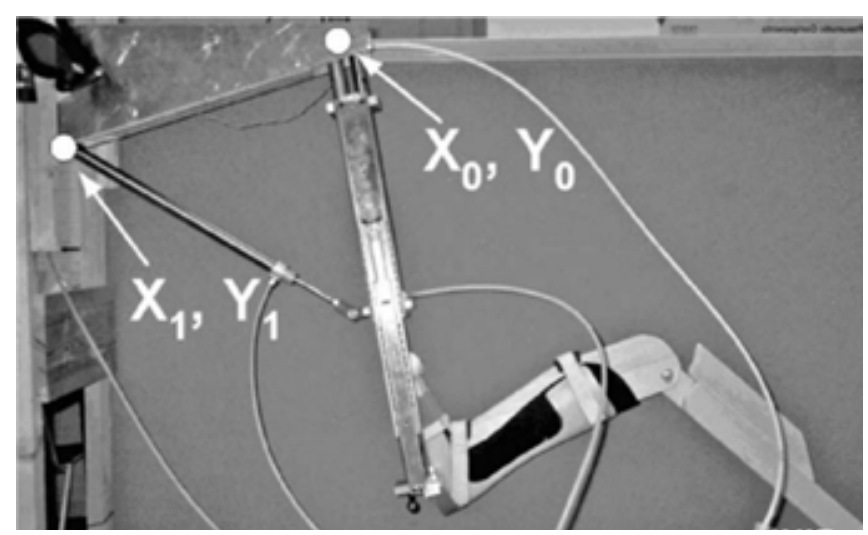

Figure 6

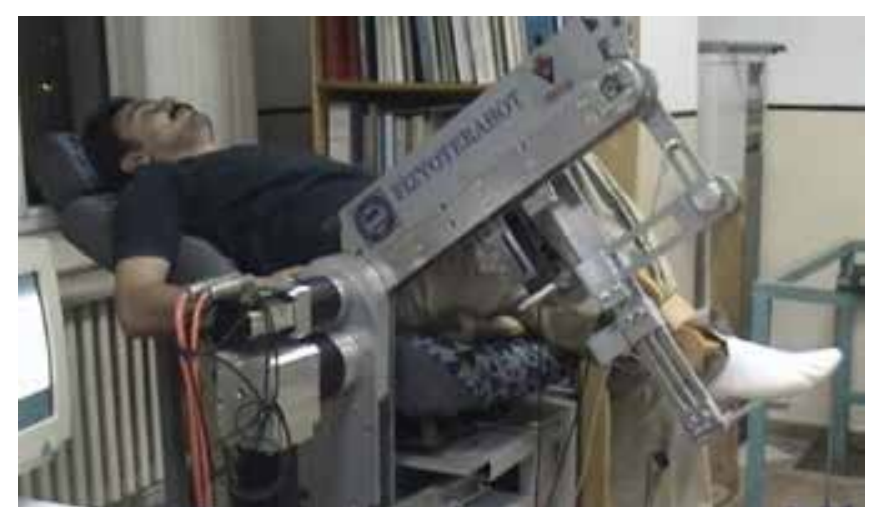

Figure 7 of the knee and hip, and unlike NeXOS, can also perform abduction-adduction movement of the hip while the patient remains seated during the exercise session instead of lying down on a table (18). Although the Human-Machine interface of this robot is well conceived and is very convenient to control the movements, the robot does not provide any tool for monitoring the physiological parameters of patients (28). Another drawback is that the robot can only be used on one leg at a time. This is more than a practical inconvenient in view of recent studies claiming that a patient can recover faster and better when the entire body is involved in the rehabilitation process (4). At the best of the authors' knowledge, no clinical trials have been carried out to test the effectiveness of this robot.

As an attempt to involve the entire body in the rehabilitation exercises, other machines have been developed and are presented in the next two sections on gait-training robots and the exoskeletons.

\section{Gait-training robots}

Gait-training robots are a class of fixed-based systems usually used in combination with a treadmill and whose original application was rehabilitation after stroke, spinal cord injury or chronic hemiplegia (see e.g. figure 8) (8). Following a series of studies suggesting that the involvement of the entire body could enhance the effectiveness of hip and knee rehabilitation process (4), the use of gait-training robots has

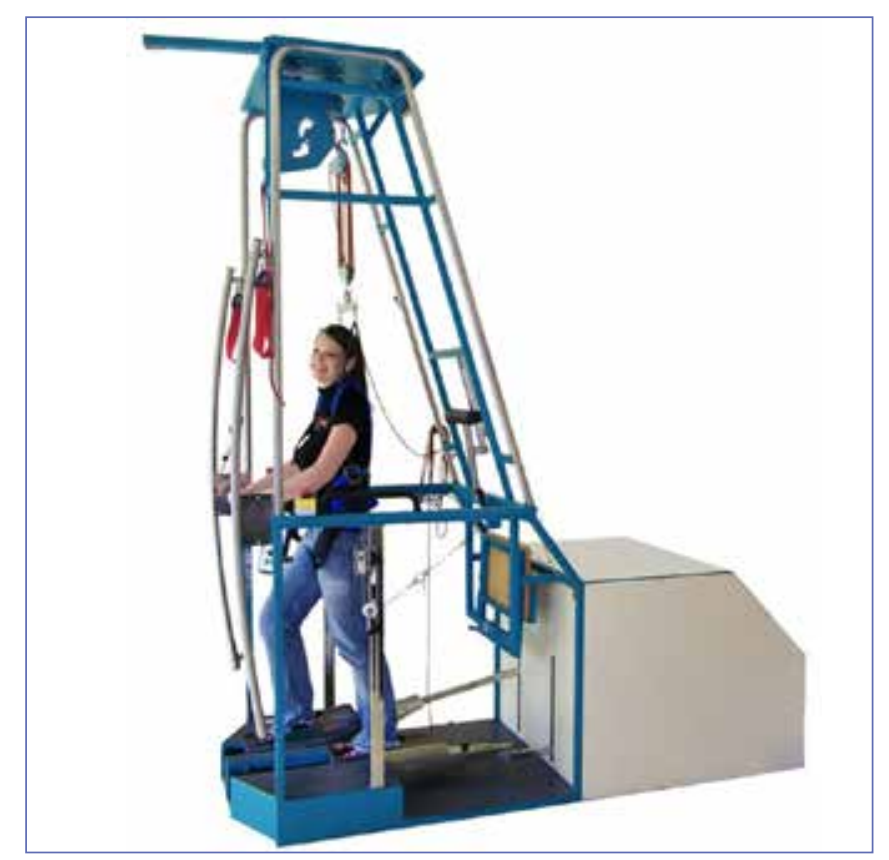

Figure 8 
recently been extended to rehabilitation after hip and knee prosthesis surgery, and for cruciate ligaments post-surgery. Three gait-training robot systems, all developed in the beginning of the 2000s, are currently commercially available and used for therapy worldwide: the gait trainer GT-I (30), the Lokomat (31), and the AutoAmbulator (32).

The Gait Trainer GT-I (figure 8) is manufactured by RehaStim, Berlin, Germany (30). It is a fixed-based structure consisting of an overhead harness unloading the patient's body weight, and two footplates driven by independently actuated mechanisms that move the feet along a fixed gait-like trajectory with a doubled crank and rocker system (figure 9). The patient is positioned using the built-in swivel device, his feet are fixed on the base plates and the wire mounts attached in order to compensate the body's side movement (30). The system is customizable to the patient physical characteristics, e.g. the stride length can be adjusted by changing gears (33). During therapy, the horizontal and vertical trunk movements of the patient are assisted in accordance with the gait phase. Meanwhile, the integrated servo drive supports the patient's own effort in order to keep the rotation speed constant, similarly to what happens in an ellipsoidal bike.

The main feature of the Gait Trainer GT-I is that it allows severely affected subjects to experience the repetitive practice of a gait-like movement without overstraining the therapists. Several clinical studies proved the efficiency of the Gait Trainer GT-I on stroke patients in comparison with conventional physiotherapy (34-36). However, Stefan Hesse et al. (33) mention that "on the gait trainer, however, one therapist should pay attention to knee motion in order to prevent knee byperextension". This risk is seemingly a consequence of the non-compliance of the Gait Trainer GT-I. Indeed, since the rotational speed is the only feedback, any resistance coming from the patient will not be taken into account by the robot. As a consequence, the robot might continue its movement even if the knee is in hyperextension. Fortunately, they also mention that "this (the knee hyperextension) can happen during the initial sessions of the therapy program; later on the patients learn to control the knee motion by themselves". In their product flyer (37) REHA-STIM also mention that the GT-I is appropriate for the treatment of a various number of diseases. The flyer claims that, thanks to GT-I, "even children with Cerebral Palsy, patients with incomplete paraplegia, Severe Head Trauma, Multiple Sclerosis and Parkinson have the opportunity to improve their ability to walk, just as well as patients with joint replacements".

However, at the current stage the effectiveness of the GT-I after joint replacements is at the best of the authors' knowledge not yet demonstrated. It is important to remark that, among the three commercial solutions mentioned above, the GT-I is the one that departs the most from the traditional therapist-assisted body-weight supported treadmill training typical of stroke rehabilitation since it interacts with the patient's lower limbs through two footplates rather than acting on the shank as human therapists do. Moreover, of the three machines, it is also the one that departs more from natural walking because the footplate principle substantially alters the sensory cues of the foot impact with the ground or treadmill band. As a consequence, it is unclear if the GT-I might provide a natural walking pattern able to respect the physiological kinematic of the knee. To the best of the authors' knowledge, there is no study focusing on this topic. The Lokomat (figure 10) is a robotic exoskeleton worn by patients during treadmill walking. Four motorized joints

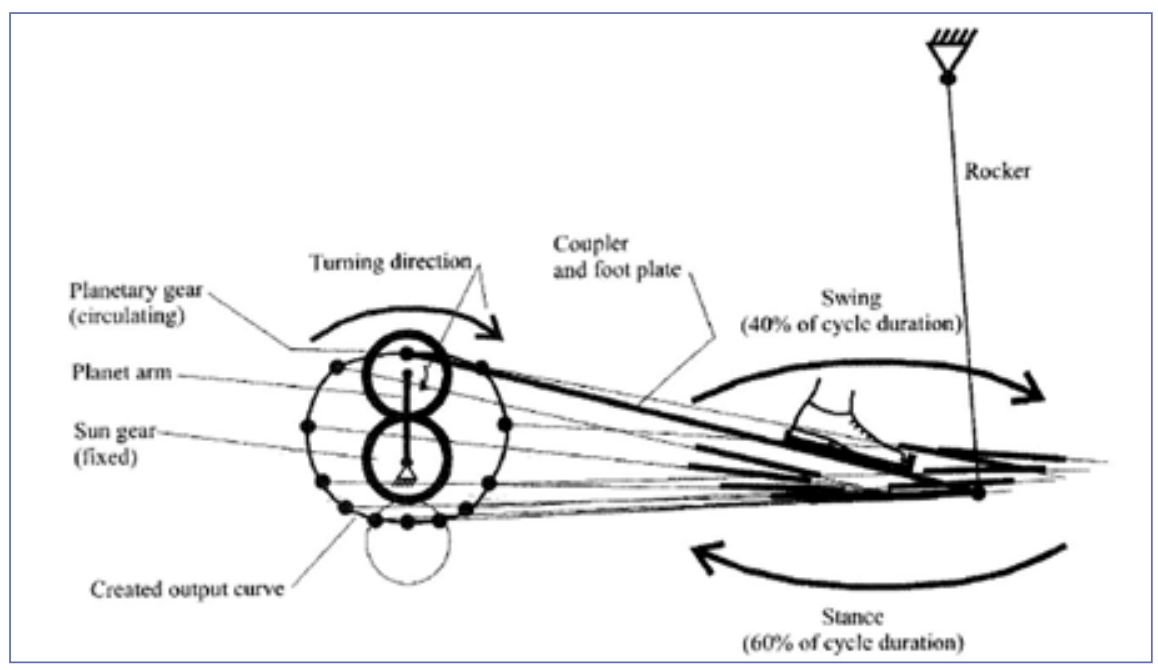

Figure 9

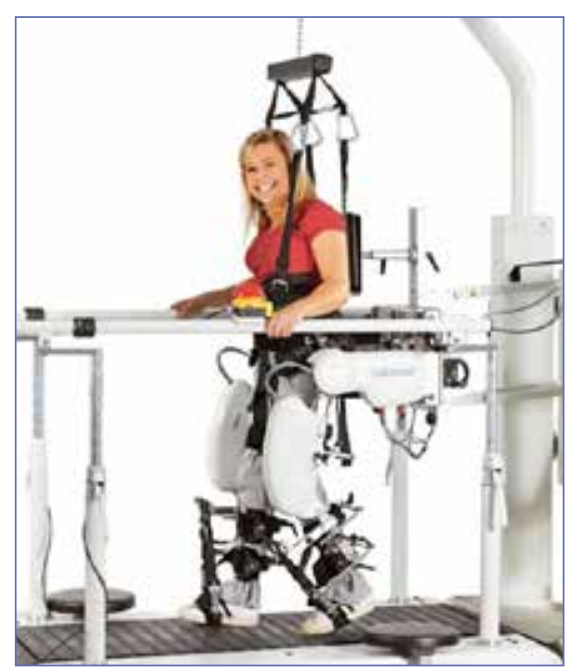

Figure 10 
(left and right hip and knee joints) embedding force sensors and driven by linear motors (two per leg) move the hip and knee of the patient. The legs of the patient are clamped to the Lokomat by one upper-leg and two lower-leg braces. A parallelogram structure that allows vertical up/down motion of the patient prevents lateral balance problems. The patient's legs are driven in a physiologic-like gait pattern along a fixed position-controlled trajectory (31), i.e. during the training the patient cannot influence the motion by himself and is likely to remain passive. The main feature of the Lokomat is the possibility to take into account the human-machine interaction and allow the patient to actively influence and adapt the gait-pattern. Indeed, it is proved that this motivates the patient and promotes greater activation and coordination of the muscles (4). Another feature of the Lokomat is the measurement of the muscular activity during the training. According to the producer (38), Lokomat is indicated for the treatment of patients with stroke, traumatic brain, paraplegia, cerebral palsy, multiple sclerosis, Parkinson's disease, endoprothesis (e.g. joints replacements), degenerative joint diseases of the lower limbs (e.g. knee osteoarthritis), spinal muscular atrophy and muscle weakness due to lack of mobility. Among the contraindications (38), it is important to remark that Lokomat training may not be conducted if there is any medical condition preventing active rehabilitation (e.g., respiratory disease, pregnancy, orthopedic conditions, cognitive deficits limiting communication, neuro-psychological conditions, infections or inflammatory disorders, osteomyelitis...), if fractures are not consolidated, or if fixed joint contractures limit the range of motion of the Lokomat orthosis. To control these risks, the legal note of Lokomat (38) advise the clinician to keep up-to-date by reading the latest scientific literature and possible changes in treatment recommendations concerning a large number of aspects, including "arthroplasty, uncontrolled hip, knee or ankle instability that would still pose a danger despite the body weight support, lack of head control, joint contractures or limitations in the range of motion due to spasticity that can be reduced, differences in leg length correctable with an insole and skin lesions (including pressure sores) in areas of contact with harness support".

Several clinical trials have been carried out to assess the effectiveness of Lokomat. In 2005, Lars Lünenburger et al. (39) used Lokomat to carry out the measurement of spasticity and voluntary muscle force on several patients with neurological disorders, using the Lokomat. This study concluded that the use of Lokomat (and of robotic devices in general) to measure the effects of rehabilitation procedures provides relevant data to assess in a quantitative way the effectiveness of physiotherapy procedures. In 2009, two contradictory studies concerning the use of Lokomat on stroke patients were published. In the first one (40), the conventional gait training interventions resulted to be more effective than robotic-assisted gait training for facilitating recovery of walking ability. In the second study (41), the results showed that the effectiveness of the rehabilitation procedure for both the robotic-assisted gait training (RAGT) group and the control group for which only manual therapy was carried out, presented no significant differences. Furthermore, self-selected walk speed, paretic step length ratio, and four of the six secondary measures were significantly higher within the RAGT group, while only balance scores were significantly better within the control group. In 2016, another clinical study (42) concluded that the capability of Lokomat of performing rehabilitation after stroke is as effective as traditional physiotherapy. In this study, the RAGT group presented higher gains in Modified Functional Ambulation Category, Modified Rivermead Mobility Index, Berg's Balance Scale, and Modified Barthel Index than the control group. At the current stage, at the best of the authors' knowledge, no clinical trials measuring the effect of Lokomat on other pathologies has been published.

The AutoAmbulator (figure 11) is commercialized by the HealthSouth Corporation, Birmingham, Alabama, USA. It

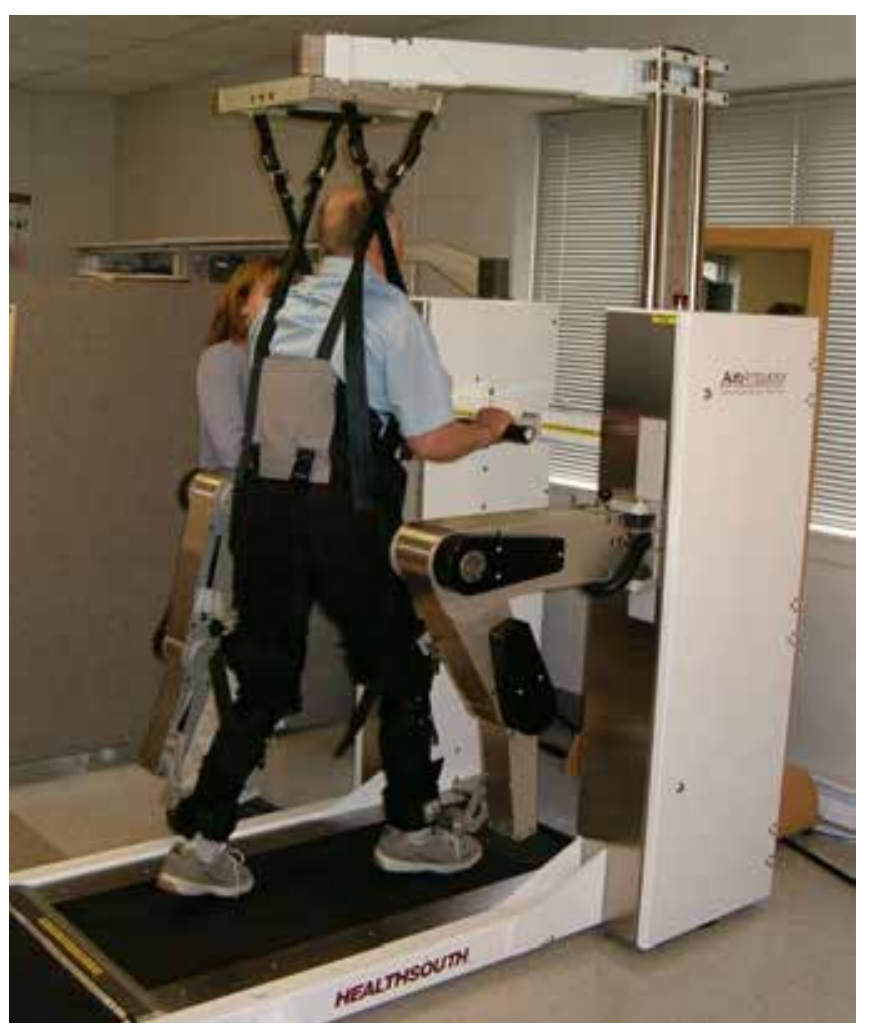

Figure 11 
consists of two robotic legs that assist patients to walk on a treadmill with their body weight supported as needed. At the current stage (June 2018) the website of the company has not been operational for several months. As a consequence, it is unclear if the AutoAmbulator is still commercialized and the available information are very limited.

Besides the commercially available robots, a number of research gait training excerpts have been proposed.

In 2003, University of California at Irvine developed its own gait-training robot "PAM and POGO" (figure 12). PAM is the acronym for Pelvic Assist Manipulator and POGO stands for Pneumatically Operated Gait Orthosis. The overall machine consists of the combination of these two devices, the five-DOF pelvic assist manipulator and the two two-DOF leg orthosis, with a treadmill and a harness to support the patient's weight. As the name suggest, PAM assist the movements of the patient's pelvis and POGO moves the lower legs of the patient thanks to two pneumatic cylinders: one providing hip flexion and extension, and the other one providing knee flexion and extension. An important feature of "PAM and POGO" is the use of pneumatic actuators costing less than electric motors and having higher power-to-weight ratios. As a consequence, the additional

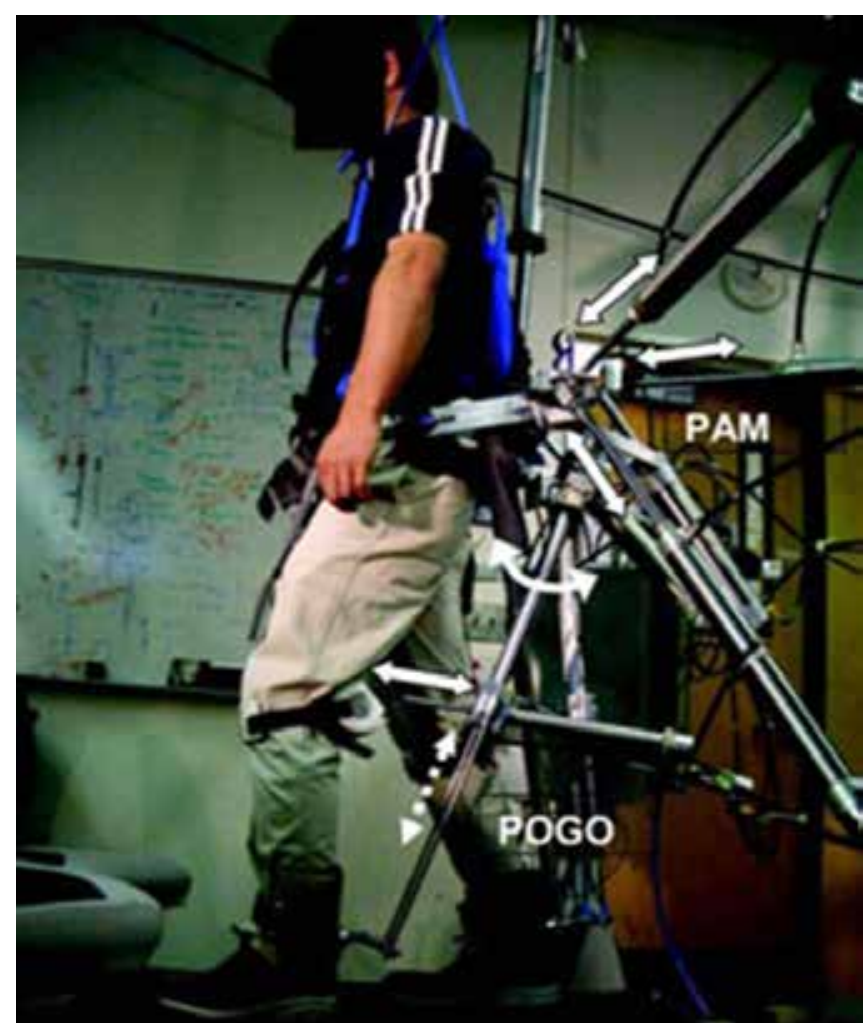

Figure 12 inertia of PAM and POGO is negligible although the robot is able to deliver high forces. The "PAM and POGO" is a compliant robot able to act as a teach-and-replay device using a system that allows the robot to move the subject's pelvis and legs following a desired motion, with or without the help of therapists.

The gait-training robot "PAM and POGO" has been specifically designed for recovery after SCIs (43). At the best of the authors' knowledge no clinical trials have been made to assess the effectiveness of PAM and POGO in a long term rehabilitation process. However, the developers Daisuke Aoyagi et al. (44) made their own experiments with spinal cord injured subjects, in order to demonstrate the safety and the performance of the basic design of PAM for assisting at the pelvis during Body Weight Support step training and to examine the capability of POGO of unloading the patient's legs. In this test, it was shown the capability of PAM to act as a motion-capture device. Indeed, PAM is able to record the pelvis trajectory, which is unique for each subject. PAM is also able to replay it repeatedly, thus providing a natural pelvic motion assistance. Interestingly, the measurements showed that the overall shape of POGO's hip trajectory, which roughly indicates the position of the ankle with respect to the hip, was similar across all the patients. The knee cylinder trajectory exhibited more variation between subjects. This can be partly due to the passive DOF around the knee cylinder. Indeed, albeit these DOF are designed to allow for natural motion and to accommodate subjects of various sizes and shapes, they also introduce ambiguity in the kinematics. In general, doing these tests, POGO provided a considerable amount of assistance, reducing the physical workload on the patients. Moreover, although misadjustment of the harness was sometimes a source of discomfort in the groin area, there was no serious discomfort inflicted by the devices. The tests also showed that POGO cannot assist in the lateral direction of the leg swing, nor prevent toe drop during swing, or control the lateral positioning of the foot landing. This is not surprising since POGO has no active control in those DOFs.

In 2004, the academic team that was behind the design of Gait Trainer GT-I developed the HapticWalker (figure 13). Similar to the Gait Trainer GT-I, the HapticWalker consists of two footplates guiding the movement of the feet. In this new design, unlike the Gait Trainer GT-I, the footplates kinematics does not depend on a double crank and rocket system but consist of two 3 DOF robot modules moving each foot in the sagittal plane. Each model consists of two linear motors moving independently on a common rail and connected via a slider-crank system that moves the robot arm along the two base axes of the sagittal plane (horizontal, vertical). Inside the robot arm, a rotary motor actuates the footplate mount- 


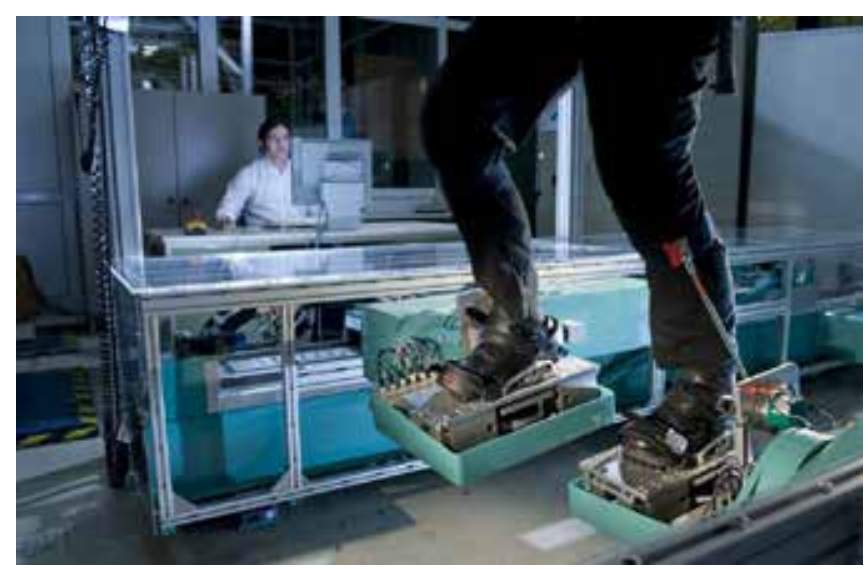

Figure 13

ed at the distal end of the arm. The foot module contains a 6 DOF force/torque sensor and the footplate. The sub-robots of this machine are designed in a modular way. In particular, it is possible to extend the capabilities of the robot by adding a second module in place of the footplate and to compose so 6 DOF robot at each footplate to enable any arbitrary rototranslation of the foot. An additional axis to support the metatarsal joint movement can also be added. The main feature of the HapticWalker is that permanent foot/machine contact is maintained during the motion. In combination with the haptic simulation of ground conditions the robot is able to reproduce different common daily life activities (e.g., stair climbing, walking, etc.). Moreover, it incorporates force feedback and compliance control (46). In 2008, a clinical study (47) presented the assessment of the quality of training sessions with the HapticWalker on a total of nine healthy subjects. The results of the trials to evaluate muscle activation in healthy subjects during free walking compared to walking on the end-effector based gait rehabilitation robot HapticWalker showed that this machine has the potential to offer an advanced gait training for Central Nervous System impaired patients. Nevertheless, all presented results cannot simply be transferred to stroke patients and further clinical trials with real patient are needed to confirm them. At the current state, just like the Gait Trainer GT-I, it is unclear that the HapticWalker might provide a natural walking pattern able to respect the physiological kinematic of the knee. To the best of the authors' knowledge, there is no study focusing on this issue nor studies concerning the use of HapticWalker on patients after knee surgery. Unfortunately, the current dimensions of this device and the fact that it requires high voltage supply are the main limitations to its clinical use (48). In 2005, Veneman et al. (49) introduced a newly developed gait rehabilitation device, called LOPES (LOwer extremity

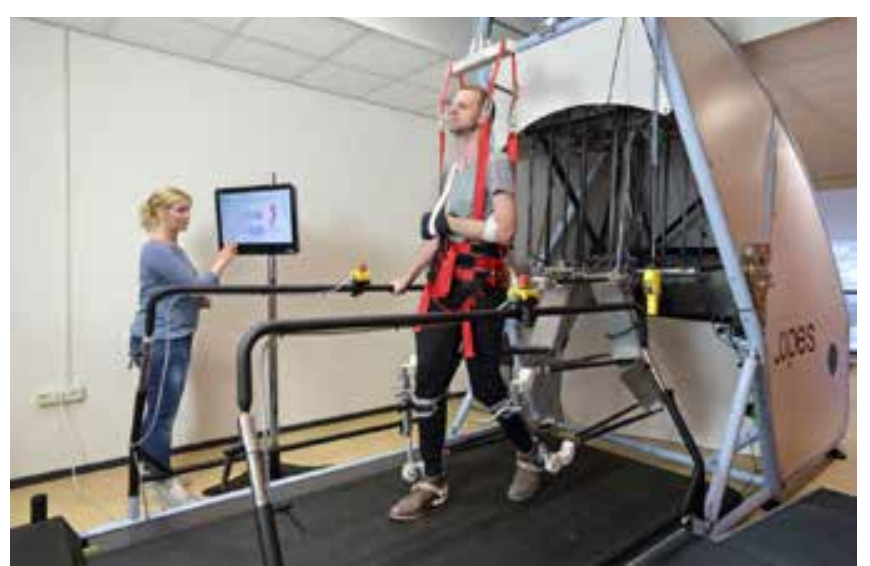

Figure 14

Powered ExoSkeleton) thought for neurorehabilitation after stroke or SCIs. Its characteristic feature lies in a freely translatable pelvis segment with a leg exoskeleton containing three actuated rotational joints: two at the hip and one at the knee. The patient walks on a treadmill and his weight is supported by the machine. In 2013, the machine was partially redesigned using a parallel structure behind the patient (shadow leg) that is connected to the patient joints with rods. The main advantage of this design is that little alignment is required, the lateral side of the hip is left free, and thus arm swing is not obstructed. Letting unhindered the arm swing is a matter of great important since it is part of normal walking and contributes to the overall stability of human gait. Moreover, the use of a shadow leg (50) allows to reduce the weight located on the patient leg itself. In 2015, the University of Twente, The Netherlands, presented the second version of LOPES (figure 14). As its predecessor, LOPES II (51) is based on the idea of the shadow leg. It has eight powered degrees of freedom (hip flexion/extension, hip abduction/adduction, and knee flexion/extension at both legs, and pelvis anterior/posterior and pelvis medio-lateral translations). All other degrees of freedom are left free, and pelvis frontal and transversal rotations are constrained. An advantage of this new design is the reduction of the donning time. LOPES II is guided by a system that converts the joint set points and desired stiffnesses to the actuator set points. The developers tested LOPES II on two healthy subjects to verify if the patient's segment angles were correctly calculated (51). They also recorded the donning time for some stroke patients and performed exploratory studies with stroke survivors and SCI patients. Both the cases of a mildly impaired stroke survivor and a severely impaired SCI patient were considered. The donning time for LOPES II was acceptable, between 10 and 15 minutes for the first 
training and between 5 and 8 minutes for the recurring trainings. For the severely impaired patients, the donning time was higher. The results also showed that LOPES II was powerful and stiff enough to enforce a walking pattern on a severely affected patient. The capability of LOPES II to provide assist-as-needed help was shown. Indeed, LOPES II was able to provide selective assistance to a mildly affected patient. The stroke survivor also showed some improvements in aspects of walking that were not assisted directly. A possible explanation is that the subjects adapt different aspects of their gait pattern, also aspects that are not assisted directly, to find a new optimal gait pattern when they receive selective support. This is mainly due to the minimal impedance of LOPES II giving the patient the freedom to adapt his gait pattern.

In 2007, Sai K. Banala et al. presented the first version of the Active Leg EXoskeleton (ALEX) (52) aiming at gait rehabilitation of patients with walking disabilities. This robot was followed in 2011 by a second version (ALEX II). Six years after the first model, the Department of Mechanical Engineering of Colombia University, New York, USA, releases the third version of the exoskeleton, the ALEX III (figure 15). The mean idea behind the design of ALEX III is the development of a more flexible gait trainer based on scientific evidence. In particular, the design was based on the consideration that the pelvis plays a fundamental role in gait: lateral pelvic motion and hip adduction/abduction are both important for load shifting between legs (53), while pelvic rotation facilitates swing (54). As a consequence, ALEX III is, to the best of the authors' knowledge, the only rehabilitation device designed to work in conjunction with a treadmill that is capable of actively controlling 4 DOFs at the pelvis

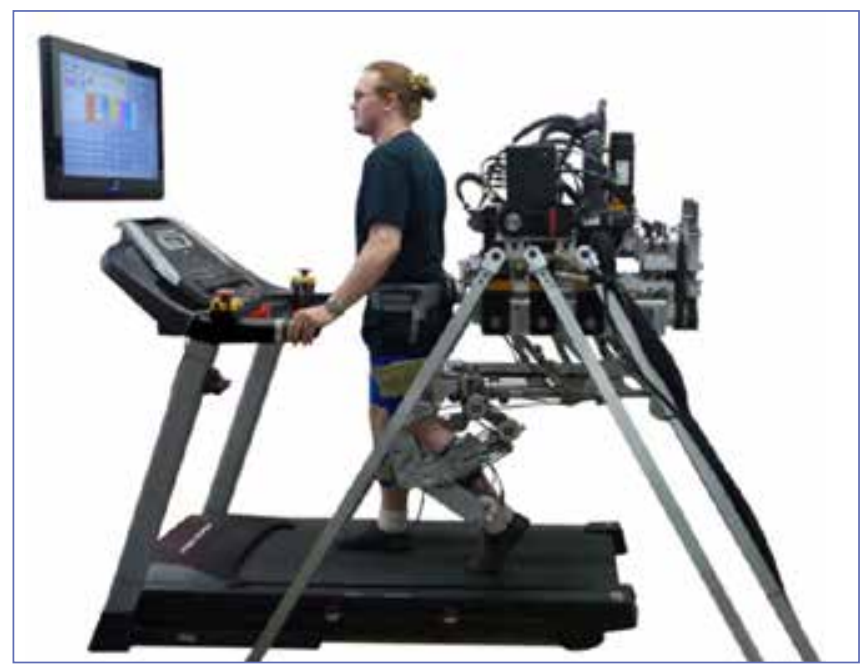

Figure 15 (vertical rotation, anterior/posterior, superior/inferior and lateral motions) and 4 DOFs at each leg (hip adduction/ abduction, hip and knee flexion/extension, ankle plantar/ dorsiflexion) (55).

The robotic leg interacts with the human leg at three interfaces: the thigh cuff (figure $\mathbf{1 6} \mathbf{n} . \mathbf{1}$ ), the shank cuff (figure $\mathbf{1 6}$ n. 2), and the ankle lever (figure $\mathbf{1 6}$ n. 3). Load cells are positioned between the cuffs/lever and the mechanical structure to estimate the interaction forces. 6-DOF forcetorque sensors are placed at the level of the cuffs, while a torque sensor measures the torque at the level of the ankle. At the current stage, torques at the hip, knee, and ankle are estimated using the load cell measurements through standard differential kinematics techniques (assuming that the cuffs and the ankle lever are rigidly attached to the human body) and are fed into separate PID regulators. In the near future the developers foresee that the legs and the platform of the ALEX III will be integrated into a single device, and a controller will be designed and implemented for the whole device. In 2017 a new haptic feedback strategy was tested on ALEX III on 32 healthy subjects (56). This control strategy, based on error enhancing, was developed to solve an issue that might occur when using assist-as-needed strategies, i.e. patients might adapt to the level of assistance, and only put in as much effort as required to complete the motion. Error-enhancing strategies increases the amount of error, hence enhance user engagement in the rehabilitation process by forcing the user to achieve predefined goal by actively resisting the device. In this study, the error-enhancing strategy is based a variable damping force tunnel and explores the effect of the shape and strength of the damping field using ALEX III. The study concludes that the explora-

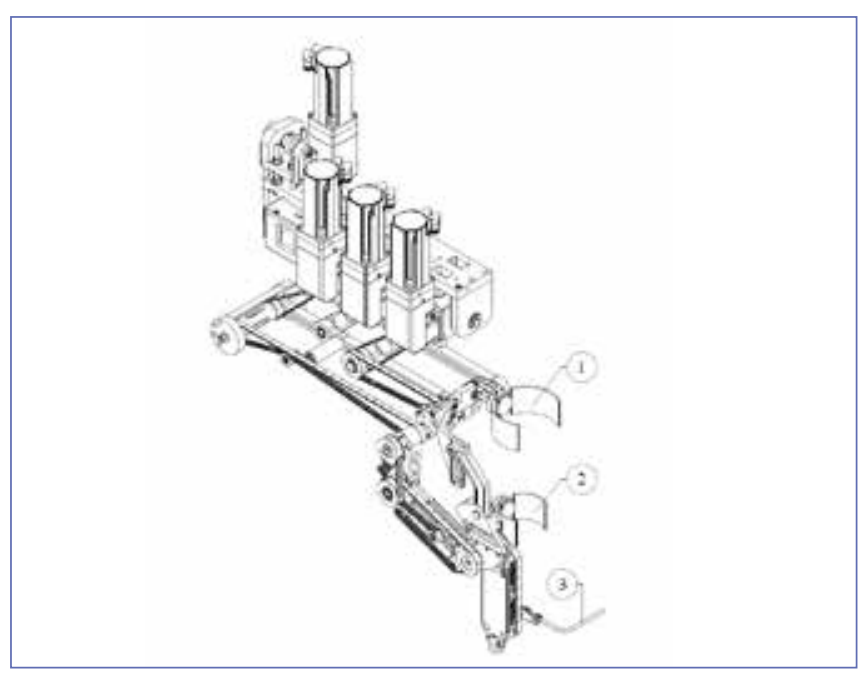

Figure 16 
tion of new types of feedback is essential to improve physical therapy, but does not allow to assess the effectiveness of the rehabilitation carried out by ALEX III.

In 2015, the same research group reviewed the entire design of ALEX III and proposed the so-called C-ALEX (57). C-ALEX (see figure 17), where C stands for cable, is based on the string-puppet principle. The objective of this design was to reduce the weight of the machine and, as a consequence, its influence on the patient leg movement and force. The use of the string-puppet principle to reduce the influence of the robot inertia was already introduced in 2004 by the String-Man robot (58). String-Man (see figure 18) consisted of a harness bearing the patient's weight, and seven wires linked to the harness, compliantly manipulating the six-DOF of the patient's torso by means of one force sensor on each wire. It should be remarked that, rather than an autonomous gait trainer, String-Man was conceived as an active pelvic support to be used in conjunction with a treadmill and the assistance of a physiotherapist, and, as such, no active actuation of the lower joint was foreseen. C-ALEX extends the concept of StringMan by adding the capability of actively intervening on the lower joints. More specifically, C-ALEX uses a series of cuffs supported by cables surrounding the waist, thigh and shin of the patient. By pulling the cables the movement is produced. Each cable is driven by a servo motor and a load cell is connected to the end of each cable to measure the tension. As a consequence, the only thing the patient has to carry is the weight of the cuffs.

The kind of rehabilitation that can be carried out using C-ALEX is not yet clearly described in the literature. The

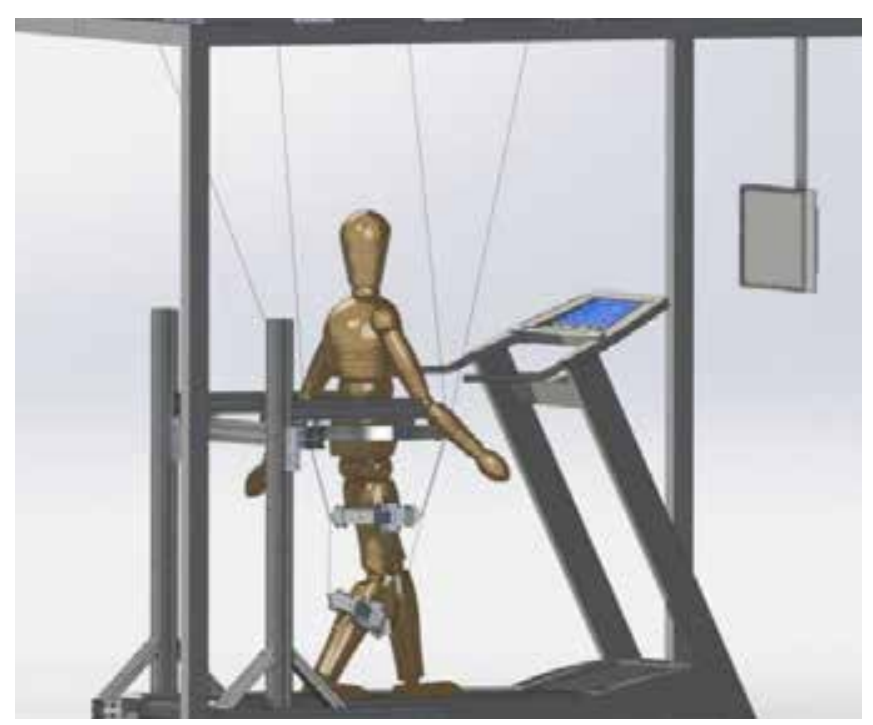

Figure 17 introduction of the paper presenting the device (57) seems to suggest that C-ALEX is mainly oriented to the rehabilitation of neurologically impaired patients. As a consequence, an "assist-as-needed" strategy has been implemented to control the robot as "The goal of the controller is to assist the ankle point of the wearer of the exoskeleton to move on a prescribed target path. The controller creates a tunnel-like force-field around the target path. If the end effector (ankle point) deviates from the target path, the controller acts as a spring and pulls the end effector back to the target path" (57).

An experiment (57) was conducted on 6 healthy subjects to evaluate the performance of C-ALEX and the results showed that C-ALEX is capable of helping the subjects to better track a prescribed ankle path.

At the current stage, the waist cuff of C-ALEX is fixed to the ground, which restricts the pelvic motion of the patient. The team has expressed the plan to solve this issue by creating a new waist cuff with a new cable routing allowing the generation of assistive hip abduction/adduction torques. The developing team also mention other limitations that will be addressed in the future such as the fact that the current cable routing can only provide assistive force in the sagittal plane and the locations of the cuffs have to be measured manually. Just like the HapticWalker, C-ALEX has a very high space volumetry, which can be a limit to its diffusion in the clinical context.

In 2011, the Pneumatically Actuated Exoskeleton (59) was designed for the gait rehabilitation of patients with gait dysfunction. More specifically, this robot consists of a 10 DOF lower limbs exoskeleton (see figure 19) positioned on

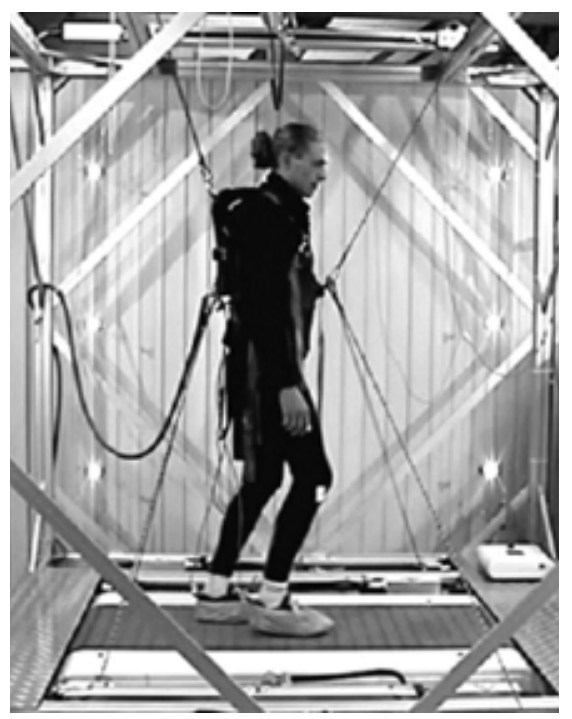

Figure 18 


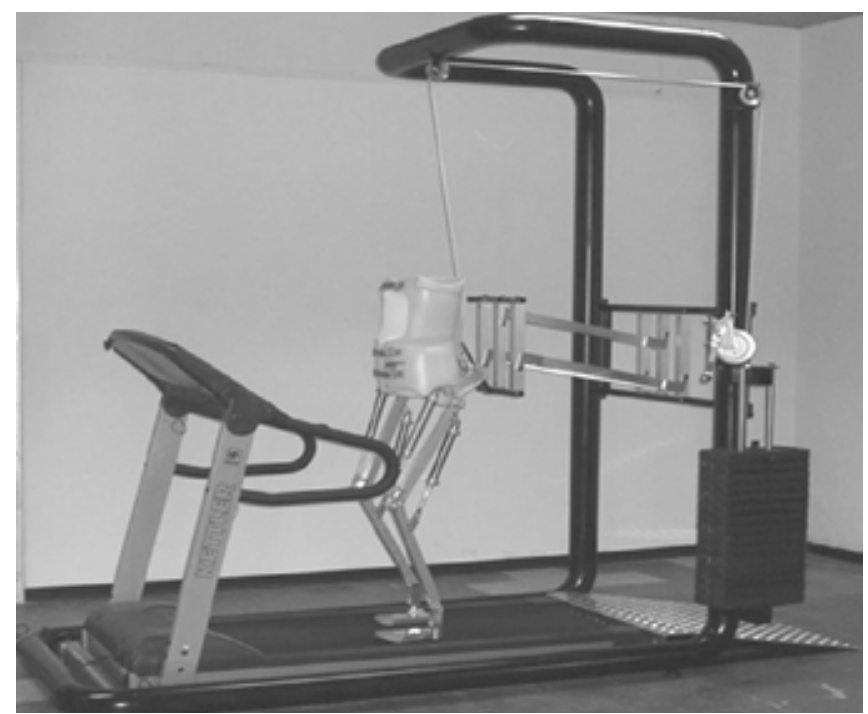

Figure 19

a treadmill and supported, at the pelvis level, with a space guide mechanism that allows vertical and horizontal movements. The space guide mechanism is connected with a fixed structure and the patient's weight is supported through a weight balance system. Hip and knee angles are measured using potentiometers and actuated by a double-acting rod pneumatic actuators (two for each leg). The control strategy is based on a fuzzy controller designed to ensure that the patient's legs will be guided along a fixed reference gait pattern. The developers foresee the implementation of a force control loop as a future development to increase the performance of this rehabilitation system. The main feature of the Pneumatically Actuated Exoskeleton is (similarly to PAM and POGO) the use of pneumatic actuators as an alternative solution to electric motors. To evaluate the performance of the exoskeleton structure together with the proposed control architecture, the developers performed experimental tests on the pneumatically actuated exoskeleton without patients. The results showed that the developed control architecture was appropriate to track any relevant trajectories. At the best of the authors' knowledge, no test on humans has been carried out on this robot.

To conclude this subsection, it is important to remark that typically trainers are constrained to a fixed-based structure (e.g. C-ALEX, ALEX III, LOPES II, etc.). As a consequence, the use of treadmills is inherent to these machines. However, although the treadmill is frequently used to simulate overground ambulation, several doubts have been raised in the literature concerning the ability of the treadmill to replicate the overground environment. Several studies (60-62) demonstrate statistically that there are significant differenc- es between overground and treadmill walking in healthy subjects for several joint kinematic and temporal variables, e.g. the stride length and the stance phase are lower in treadmill walking than overground walking. A possible explanation is that some subjects are more anxious when walking on a treadmill. Another study (63) demonstrated that a lack of optical flow during treadmill walking can contribute to significant differences between overground walking and treadmill walking. Despites the drawbacks, treadmill walking has many advantages. It allows to record ground force reactions at constant gait speeds, the kinematics, electromyography, and rate of oxygen consumption simultaneously and the space treadmill requires is by far smaller than the space required for overground walking. At the current stage, it is unclear if the use of a treadmill affects negatively the rehabilitation process.

\section{Exoskeletons}

Exoskeletons are a class of wearable robots originally called extenders (11), because they were meant to extend the strength of the wearer beyond its natural abilities. Exoskeletons consist of several rigid links assembled in a structure whose distinctive characteristic is that its kinematic chain maps into the human limb anatomy, i.e. each human anatomical joints has a one-to-one correspondence with the robot joints or sets of joints. Each joint might be actuated or not, along one or more directions and/or rotations. Exoskeletons can be seen as wearable robots providing an ergonomic human-robot interface which allows an effective transfer of power between the wearer and the robot (7).

Several kinds of exoskeletons exist. Depending on the application field, powered exoskeletons can be designed for military purposes (e.g. HULC, see figure 20), to assist and protect construction workers (e.g. the Power Loader Light of Panasonic, Osaka, Japan (64)), to aid the survival of people in dangerous environments (e.g. radioactive contaminated regions), to ensure citizens self-defense (e.g. Powered Jacket MK3, see figure 21), and to promote mobility assistance and rehabilitation for aged and infirm people (10).

The story of exoskeletons began in the early 1960s (10) when the U.S. Department of Defense funded General Electric to develop the first exoskeleton prototype, Hardiman (see figure 2). Hardiman was intended to allow the wearer to lift weights up to $682 \mathrm{~kg}$. The history of Hardiman was quite troubled, and by 1970 only one arm was completed and, although it could lift $341 \mathrm{~kg}$ and satisfied most of the functional specifications required, it weighed more than $500 \mathrm{~kg}$.

Despite these not promising beginnings, in the last 50 years military research has constantly invested in the exoskele- 


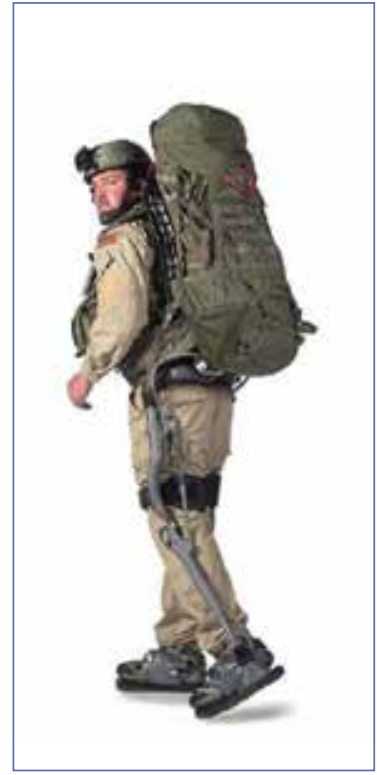

Figure 20

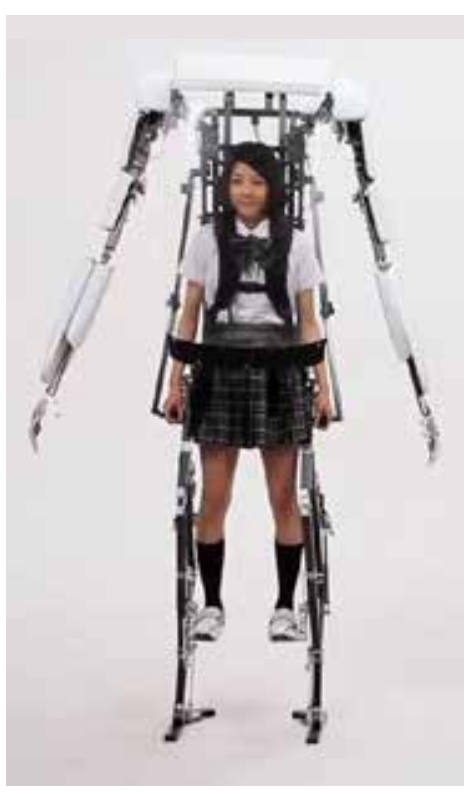

Figure 21

tons technology and a large number of exoskeletons has been developed for military purposes, such as HULC (2008) (67) and Raytheon XOS 2 (see figure 22). The main goal of military exoskeletons is to amplify the power of the wearer: thanks to force and motion sensors, the computer interface monitors the user's gestures and motion, and uses this information to interpret the intention of the user and translate it into action. HULC enables soldiers to carry loads up to $91 \mathrm{~kg}$. The weight of the load gets transferred to the ground through the links of the exoskeleton and it is powered through lithium polymer batteries. Raytheon XOS 2 is powered by an internal combustion engine and allows the soldier to lift $22 \mathrm{~kg}$ with each arm (68).

The first recorded use of exoskeleton in medical assistance dates back to the development of HAL, the Hybrid Assistive Limb exoskeleton (69). HAL was designed to train doctors and physical therapists, assist the disabled, help workers at carrying heavy loads, and aid in emergency rescues. The research works began in 1992 at the University of Tsukuba, Japan, and a first prototype was built in 1997. HAL-2, HAL-3 and HAL-5 followed, each including an improvement on the predecessor (70). HAL weighs $23 \mathrm{~kg}$ and is actuated by electric motors powered by a 100 $\mathrm{V}$ AC battery able to last for up to $3 \mathrm{~h}$, depending on the usage. Unlike many military robots like Raytheon XOS 2, HAL does not detect the wearer's motion but uses sensors directly detecting the myoelectric signals on the skin, i.e. the voltages associated with the brain signals sent to the muscles. This results in a faster response. A further advan-

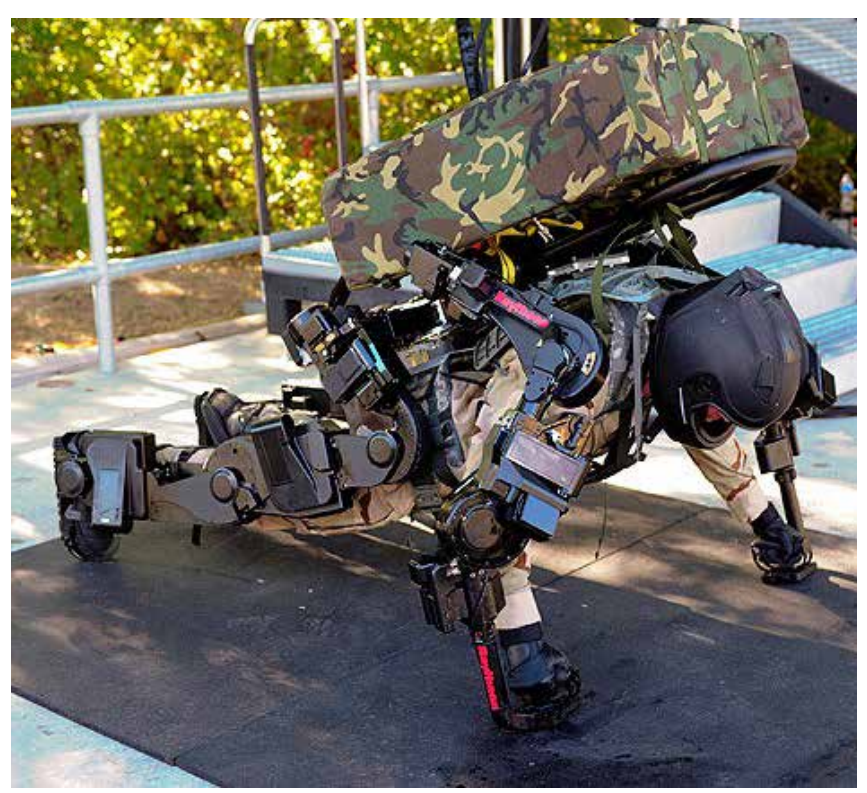

Figure 22

tage of myoelectric sensing is that it allows people suffering from spinal cord injuries or with paralyzed limbs to use the suit (65). In 2004, the developers established their own company called Cyberdyne, Tsukuba, Japan and started to commercialize the HAL series.

Later on, the huge potential of exoskeletons to assist patient rehabilitation after injuries has attracted the attention of researchers and manufacturers. As a consequence, new exoskeletons specifically designed for rehabilitation purposes have been studied and developed. Among these exoskeletons, it is sometimes difficult to distinguish the assistive from the rehabilitative purpose, because it is precisely the assistance brought to the patient that activates the sensorimotor functions and implicitly stimulates their regeneration. In other words, a simple assistance is sometimes enough to start the rehabilitation process.

At the best of the authors' knowledge, six exoskeletons specifically designed for assistive and rehabilitative purposes are currently available on the market: HAL (69), Ekso GT (71), REX (72), ReWalk (65), Indego (73) and Phoenix (74). The development of Ekso GT started in 2010 when Berkeley Bionics introduced eLEGS (see figure 23). eLEGS stands for "Exoskeleton Lower Extremity Gait System" and allows paraplegics to stand and walk with crutches or a walker, as well as turn, sit down, and stand up unaided. The design of eLEGS also allows the users to easily put on and take off the device by themselves. In 2011, Berkeley Bionics changed its name to Ekso Bionics, Richmond, California, USA and eLEGS was renamed Ekso (see figure 


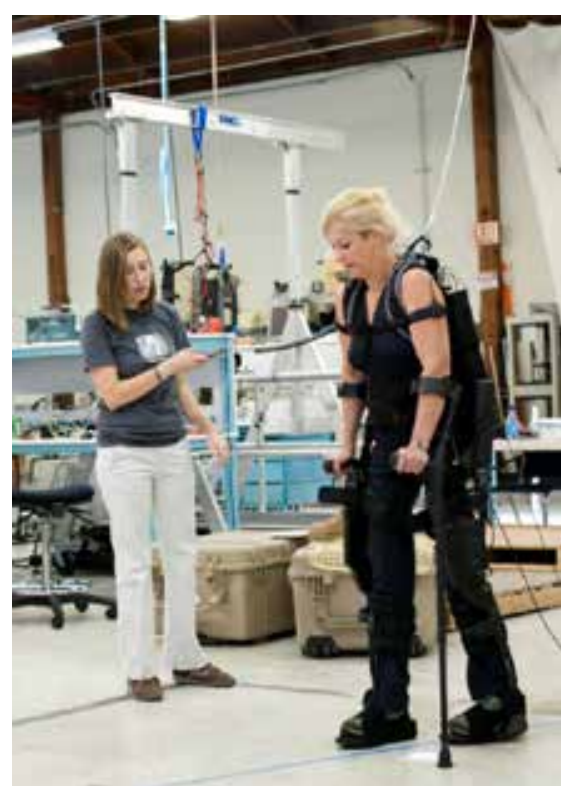

Figure 23

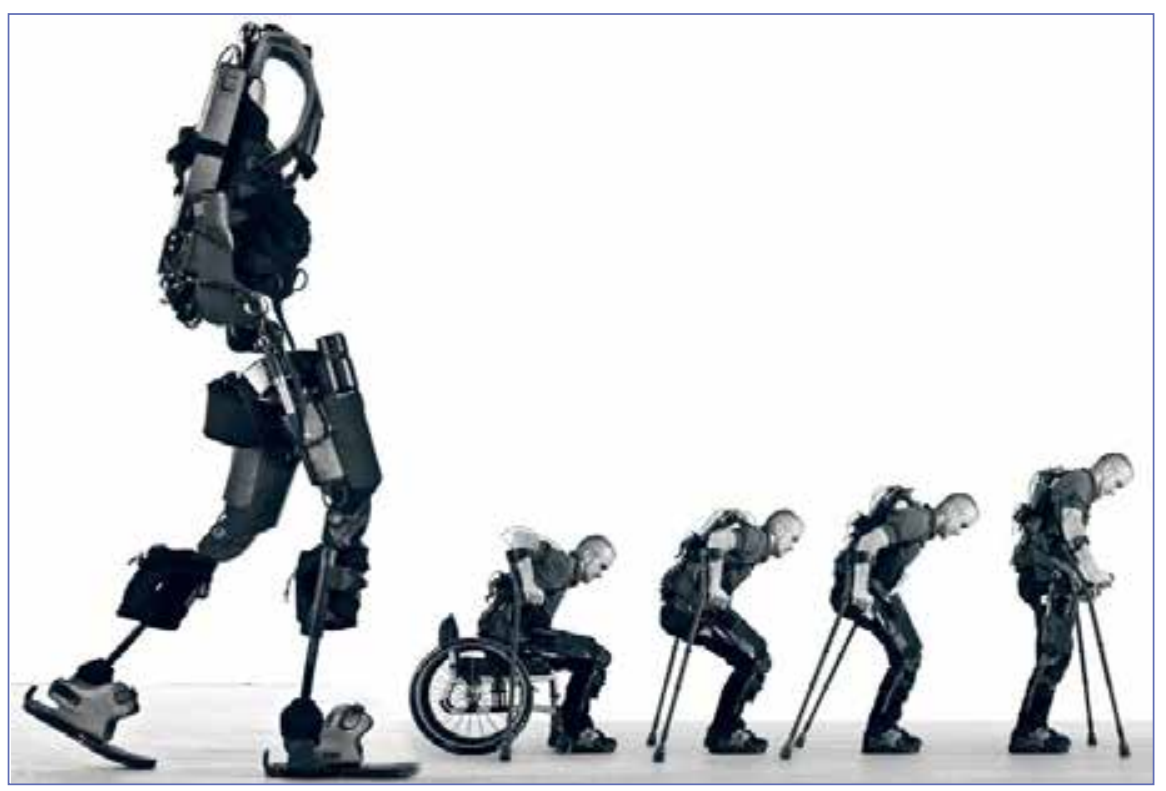

Figure 24
24). In 2013 a new version of Ekso called Ekso GT was released by Ekso Bionics. Ekso GT allows hip abduction/ adduction, hip flexion/extension, knee flexion/extension, and ankle dorsiflexion. The main feature of Ekso GT is the combination of the exoskeleton with a functional electrical stimulation (FES) technology. FES technology allows to artificially generate body movements in individuals who are paralyzed. The product website (71) mentions that the combination of both the FES with the exoskeleton enhance movement and gait training in the rehabilitation of stroke patients. Ekso GT is made of aluminium and titanium. It weighs $23 \mathrm{~kg}$ but transfers all its weight through the structure onto the ground. It initiates steps through electric motors and can be controlled either by a physiotherapist pushing buttons behind the wearer or by the wearer himself through the use of crutches. Ekso GT includes a gyroscope, trajectory sensors, and torque sensors, to detect the level of assistance each user needs and to know how much assistance to provide for every single step. The device costs over $\$ 100,000$ per unit but many patients are able to access them in clinics, "via charity or medical legal cases" (75). A limited amount of publication about the functioning of Ekso is available. Several patents (76)-(78) about exoskeletons have been submitted by Berkeley Bionics and Ekso Bionics. In (76), the exoskeleton is described as a trunk structural element connected to two leg supports at respective hip axes, allowing only hip flexion/extension. Each leg support consists of a thigh link and shank link connected at the knee joint by a single pivot joint allowing only knee flexion/extension. The knee and optionally the hip are actuated. In (77), "a first aspect of the invention concerns an exoskeleton system wherein one or more of the joints, or entire exoskeleton appendage, are modular in construction such that different versions of the joint, or exoskeleton appendage, can be installed on the fly by the end user on a single exoskeleton device". In fact, there are 4 versions of the leg, two actuated legs (right and left) and two unactuated legs (right and left). The legs are articulated at the hip, knee and ankle. The hip joint consists of a combination of two pivot joints enabling both hip abduction/adduction and flexion/extension. The knee joint is a single pivot joint allowing flexion/ extension and an ankle pivot joint connects the shank link to the foot link. All pivot joints may include orientation sensors in communication with the exoskeleton controller. Both actuated and unactuated legs share most of the same feature except for the knee. Indeed, the actuated leg includes a different knee configuration containing an electromechanical brake system.

In 2016, Ekso Bionics received the first FDA clearance for the use of Ekso GT as a rehabilitation tool for patients with stroke and spinal cord injury at levels up to C7. A Clinical study (79) assesses the safety and feasibility of using Ekso GT to aid ambulation after SCI. This study concludes that Ekso GT is safe for patients with complete thoracic SCI in a controlled environment, in the presence of experts, and may eventually enhance mobility in those without volitional lower extremity function. 

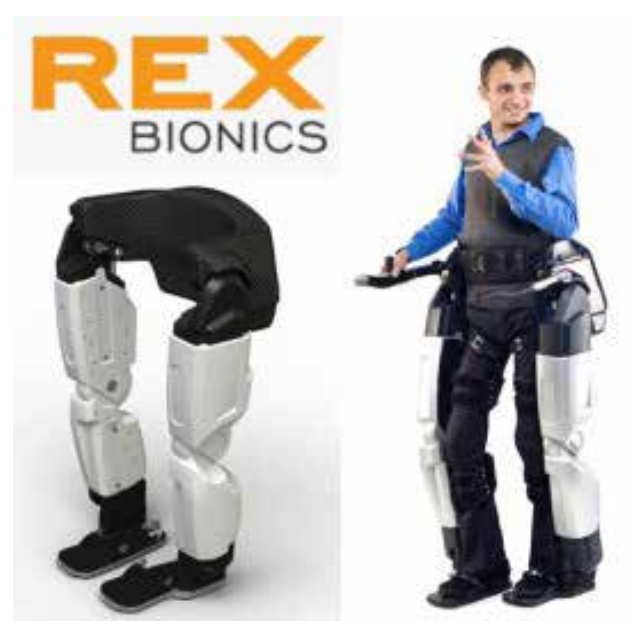

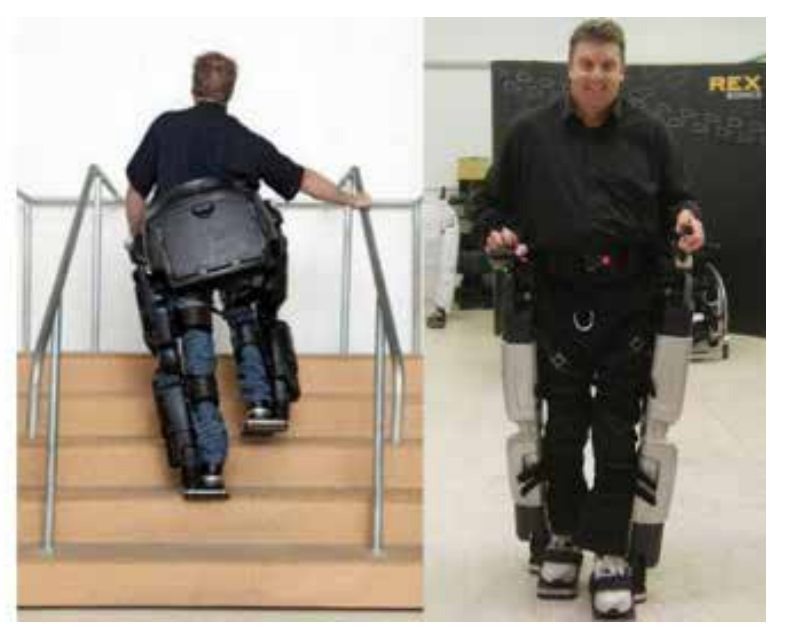

Figure 25
In 2011, one year after the release of eLEGS, Rex Bionics, Auckland, New Zealand, unveiled REX (see figure 25) (72), a lightweight, compliant and yet powerful exoskeleton enabling people with mobility impairments to walk, make turns, and even climb stairs and slopes without crutches. REX (81) consists of a rigid pelvic support (see figure $26 \mathrm{n} .1$ ) and two leg structures each consisting of an upper leg structure (see figure $\mathbf{2 6}$ n. 2), a lower leg structure (see figure $26 \mathrm{n} .3$ ) and a foot structure (see figure 26 n. 4). The rigid pelvic support and the upper leg structure are connected at the hip joint by a rose joint (see figure $26 \mathrm{n}$. 5) allowing both flexion/extension and adduction/ abduction. The upper and the lower leg structures are connected at the knee joint by a single pivot joint (see figure 26 n. 6) allowing flexion/extension. The lower leg and the foot structures are connected at the ankle joint by a rose joint to facilitate its multi-axis pivoting capability. REX has 5 DOFs at each leg actuated by a main hip actuator (see figure $26 \mathrm{n} .7$ ), a knee actuator (see figure $26 \mathrm{n} . \mathbf{8}$ ), a main foot actuator (see figure $26 \mathrm{n} . \mathbf{9}$ ), a secondary hip actuator (see figure $26 \mathrm{n} . \mathbf{1 0}$ ), and a secondary foot actuator (see figure $26 \mathrm{n} . \mathbf{1 1}$ ). The actuators are low voltage DC linear actuators whose position is measured by a sensor located inside the actuator. REX is designed to work with users of different heights, i.e. the size of the upper and lower leg structures is adjustable. REX weighs about 38 $\mathrm{kg}$, can assist a user of up to $100 \mathrm{~kg}$ and is a little bit slower than eLEGS. To control REX, the user moves a joystick located on one of REX's arms. The main feature of REX is the use of series elastic actuation and parallel elastic actuation to emphasize the mechanical compliance of the robot and to be able to provide enough power with electrical actuators during the force peaks. A clinical trial (82) states that exercising patients with multiple sclerosis (MS) in the
REX Robotic Exoskeleton System may improve posture with resulting benefits for the patient. On the product website, a case report (83) assesses the capability of REX to improve the quality of life of a 35 -year-old patient with

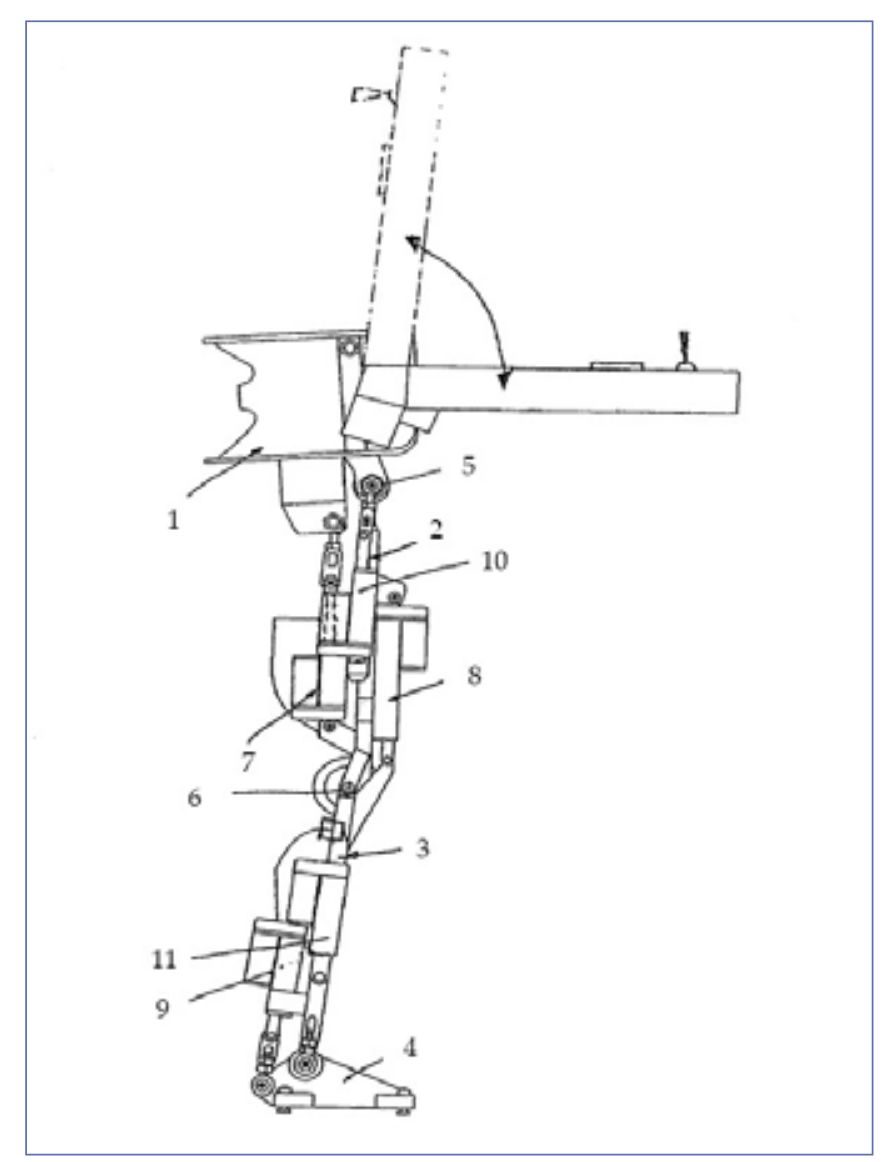

Figure 26 
a SCI. This study showed significant improvements in the participant's quality of life and a perception of pain in the neck, shoulders, and upper thoracic spine; as well as a complete elimination of headaches.

ReWalk (65) (see figure 27) enables wheelchair users with lower-limb disabilities to stand, walk, and even climb stairs. ReWalk consists of DC motors at the hip and knee joints, rechargeable batteries, and a set of sensors analyzing the position of the wearer. The user controls ReWalk by changing his position, i.e. upper-body motions are analyzed and used to trigger and maintain gait patterns and other operations, e.g. climbing stairs and shifting from sitting to standing, leaving the hands free for self-support. For stability and safety, the user also uses crutches. The official website (84) states that ReWalk is the first commercially upright walking assistance tool receiving FDA Clearance (2011). Several clinical trials (e.g. (85)-(87)) proved the effectiveness of ReWalk on patients with paraplegia or with SCI.

Indego (see figure 28) is a powered lower limb exoskeleton enabling people with spinal cord injuries to walk and

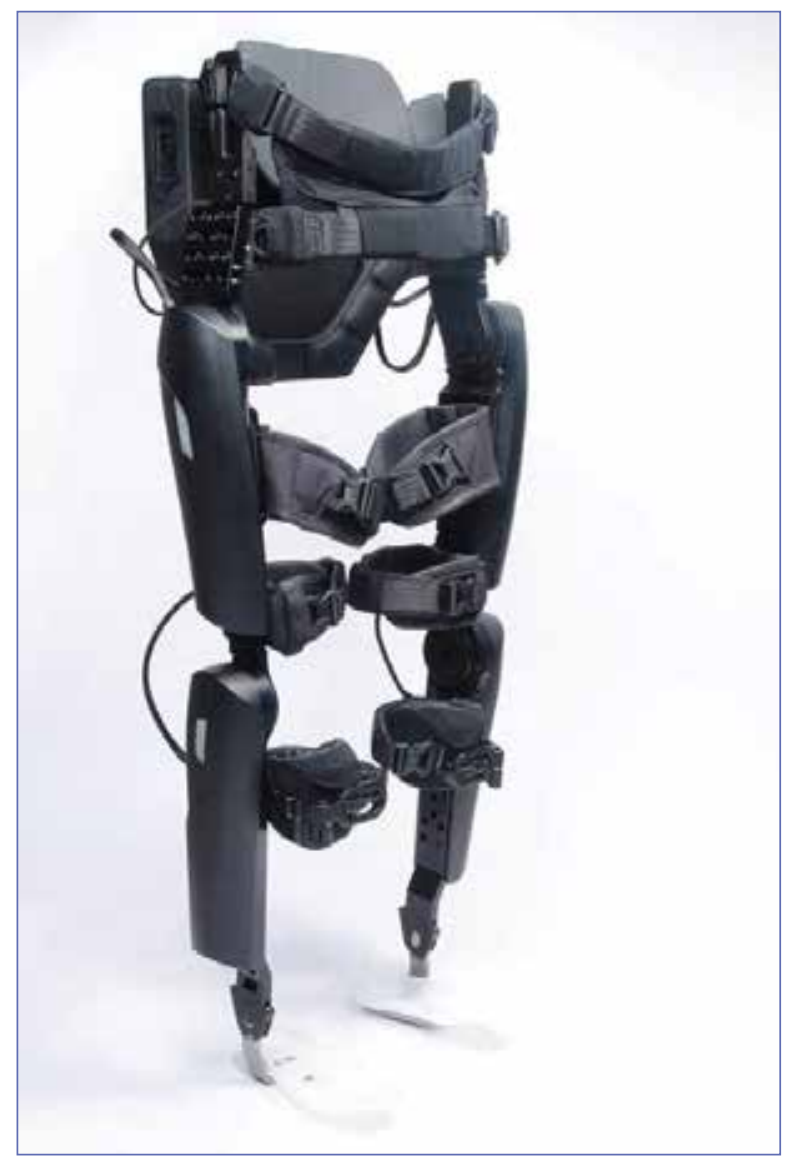

Figure 27 participate in over-ground gait training. Indego consists of six segments easy to assemble and including vibratory feedback, LED indicators, and a wireless software allowing the segment to communicate with one and another. It is actuated at the hip and the knee by electric motors. The user controls Indego by changing his posture, i.e. lean forward to stand and walk, maintain an upright stance to stop, and lean backward to sit. Indego weighs only 12 kilos, is able to carry out $113 \mathrm{~kg}$ and costs $\$ 80,000$. It received FDA Clearance and CE Mark, allowing it to be sold commercially in the U.S. and Europe since November 2015. The official website (64) reports its use in several "leading rehabilitation centers" demonstrating the benefits of Indego in therapy and at home. A recent clinical study (88) is currently ongoing to assess the use of Indego as a gait training tool for subjects with complete or incomplete paraplegia as a result of SCI who have preserved lower extremity function. The study hypothesized that subjects with complete or incomplete paraplegia who have preserved their lower extremity function will experience functional improvements after gait training with the Indego exoskeleton. At the current stage (June 2018), the results of this study are not yet available.

The Phoenix exoskeleton (see figure $\mathbf{3 0}$ ) is commercialized by SuitX, Berkeley, California, USA, a company that has spun off the Berkeley's Robotics and Human Engineering Laboratory, and whose founder and CEO is the mechanical engineering professor Homayoon Kazerooni. Kazerooni and his team have developed a series of exoskeletons over the years. Their work in the field began in 2000 with the development of the military exoskeleton HULC. In 2011

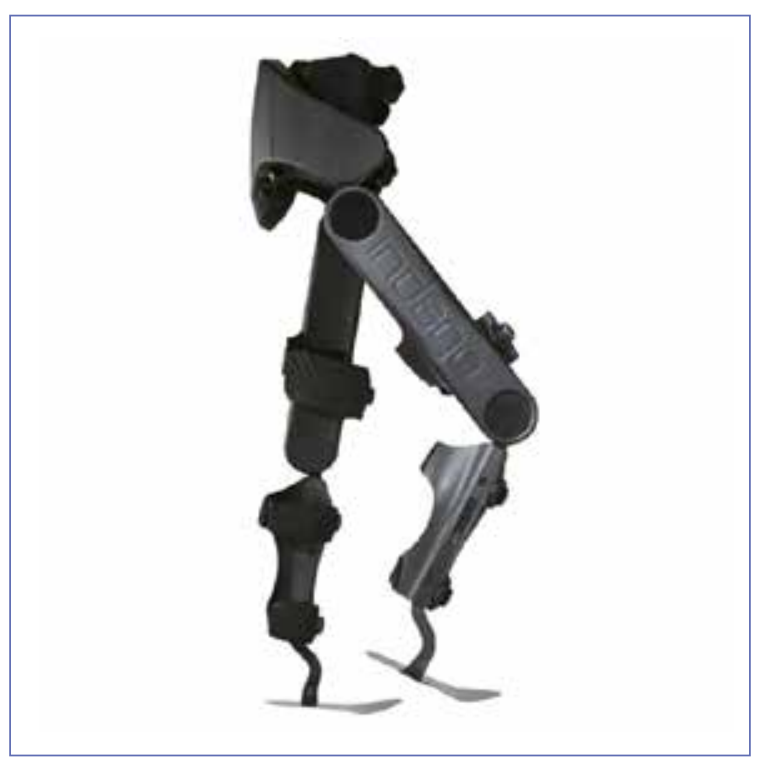

Figure 28 
they introduced Austin (see figure 29), a low cost exoskeleton for individuals with mobility disorders (89), whose main purpose was to research new technologies to create reliable and inexpensive exoskeleton systems for everyday personal use. Today, the Phoenix is one of the lightest and most accessible exoskeletons in the market. It has been designed to help people with mobility disorders to be upright and mobile. Phoenix has only two actuators at its hip. Unlike Indego, SuitX is not actuated at the knee joints, but the knee joints are designed to allow support during stance and ground clearance during swing. Similarly to Indego, Phoenix is a modular exoskeleton allowing the user to independently put on and remove each piece. Phoenix is slightly heavier than Indego, as it weighs $12.25 \mathrm{~kg}$. As stated in SuitX website (74), "In the clinic, at home, and in the workplace, Phoenix has successfully enabled many individuals to stand up, walk about, and speak to peers eye-to-eye". At the best of the authors' knowledge, there is no clinical study available concerning Phoenix albeit the official website (74) encourage persons with SCI to enroll for a pilot test. At the current stage, the developers applied for the FDA approval. Besides the commercially available products a large number of exoskeletons has also been developed in the academic field. Similarly to the gait trainers, an important research topic about the exoskeletons has been the miniaturization of the mechanical components, in particular the actuators, without sacrificing power in order to minimize their influence (e.g. the mass and the inertia) on the wearers.
In 2009, the Tibion PK100 (see figure 31) was designed to help patients affected by neurological conditions including stroke, multiple sclerosis (MS), and Parkinson's disease (90). In contrast with the typical lower limbs exoskeletons, the Tibion PK100 is a bionic leg orthosis, i.e. its use is limited to only one leg. The main feature of Tibion PK100 is its compact design which reduces its influence on the leg. The exoskeleton structure is made of carbon fiber and is actuated only at the knee joint. The active elements are located in a housing attached to the orthosis and positioned over the thigh. The housing supports and protects the actuator, battery, electronics, and user interface. A foot sensor inside the shoe provides the weight-on-foot information used by the control algorithms in order to determine when the patient requires extra assistance for sit-to-stand or stair ascent. Other sensors provide information about the knee angle, the force applied by the actuator, internal temperatures, motor currents, and battery voltage. Tibion PK100 has three operative modes (AUTO mode, CPM mode, RT mode). The AUTO mode assists the motion made by the patient. The CPM (Continuous Passive Motion) mode provides the ability for the Tibion PK100 to apply forces in both the extension and flexion directions for slow repetitive motion of the leg. The RT mode, standing for Robotic Therapy, is a stationary therapy regimen in which the actuator assists in completing a motion when the patient slows down or cannot complete the motion by himself. A preliminary study on three post-stroke patients was completed at the

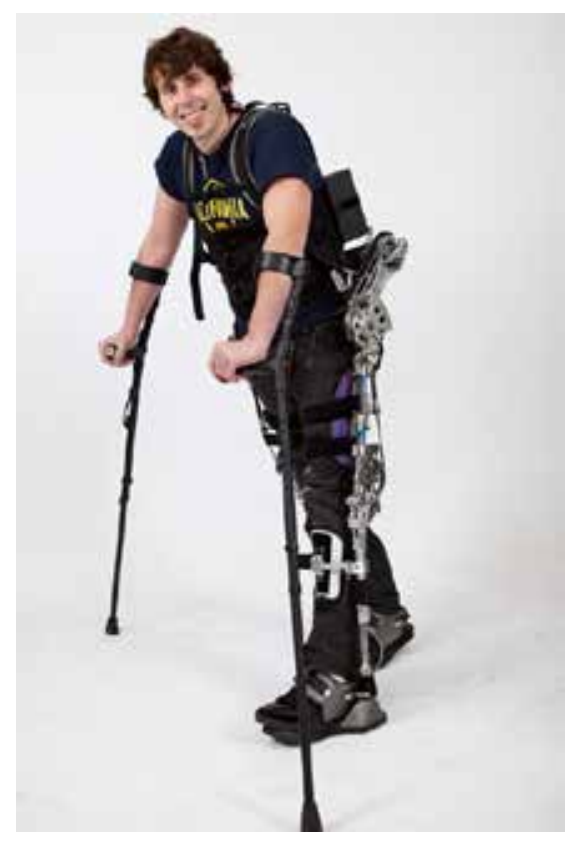

Figure 29

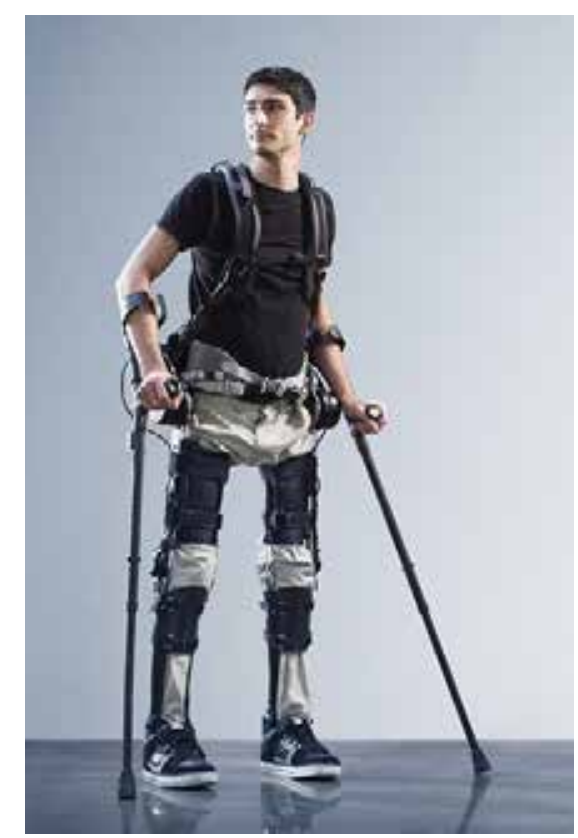

Figure 30

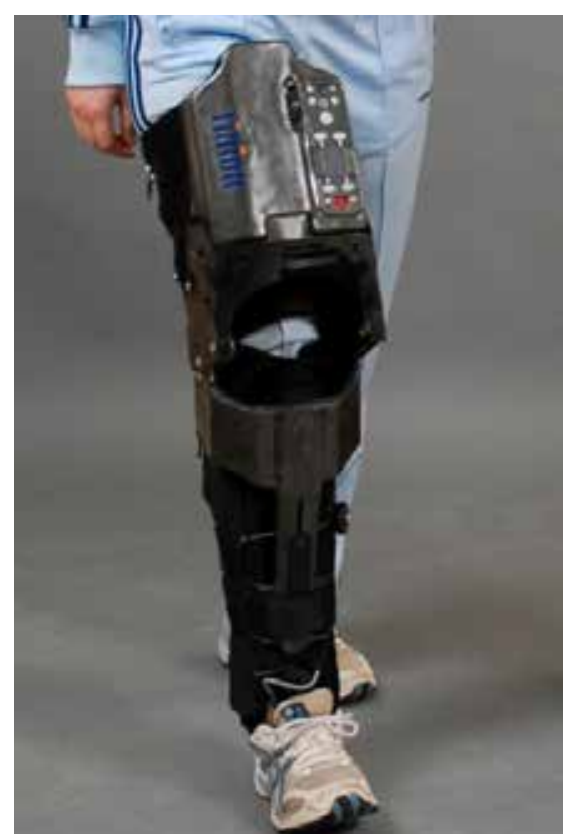

Figure 31 
UCSF Department of Physical Therapy and Rehabilitation Science. At the end of the study, all three patients achieved significant improvements in walking speed.

The same year (2009), the NASA unveiled NASA-IHMC X1 Mina (see figure 32), a robotic device developed for a range of applications including mobility assistance for abled and disabled users, rehabilitation, and exercise (91). X1 Mina has 10 DOF (see figure 33), five per leg (hip pitch, hip roll, hip yaw, knee pitch and ankle pitch). Hip pitch, hip roll and knee pitch are actuated, and the two others (hip yaw and ankle pitch) are passive DOF. The joints on each leg are connected in series, starting at the hip and going down to the ankle (92). The links of the exoskeleton can be adjusted to the user's body size such that the powered joints are co-located with the user's joints. Position and force sensors are located on the actuators. In addition, foot switches detect if the foot is on the ground. There are two switches per foot: one at the heel and one at the toes. The foot switches enable the control system to determine whether the exoskeleton is on one foot or on both feet. In addition, X1 Mina has two control mode: a torque/force control mode and a position control mode. In torque control mode the actuator attempts to apply a torque equal to a desired torque. In position control mode the exoskeleton positions the user's legs, moving them through a pre-programmed motion. The control system can also mix the two control modes to conform to the user's specific conditions (e.g. a patient paralyzed in only one leg). The main feature of X1
Mina is the use of Series Elastic Actuators. In a Series Elastic Actuator, a compliant element is placed in series with the actuator output, and the force is computed by measuring the compression of the compliant element. The advantage of this system is that it gives very accurate force feedback and has a low impedance, albeit it has a relatively low bandwidth at high forces. A study (93) was conducted by the developers to evaluate X1 Mina on two paraplegic patients. The results of this study highlight some of the areas for improvement of the mechanical design, in particular to improve the donning time. In (93), the developers also foresee the potential to use X1 Mina as part of a gait rehabilitation intervention for spinal cord injured patients. At the current stage, at the best of the authors' knowledge, there is still no clinical trials to assess this intuition.

In 2011, several research groups (94-96) brought an important contribution to the development of exoskeletons in particular, and of the rehabilitation robots in general, by highlighting a well-known fact in biomechanics literature: "Misalignment of joint axes causes detrimental parasitic forces on the patient at the attachment points and at the joints, resulting in discomfort, pain or even long term injury under repetitive use. Most crucially, axis misalignment promotes compensatory movements that can inhibit recovery and decrease real life use of the limb due to unfavored energetics of these movements". (97).

Misalignment causes are multiple, e.g. the elasticity of the human soft-tissues to which rigid part of the robot are

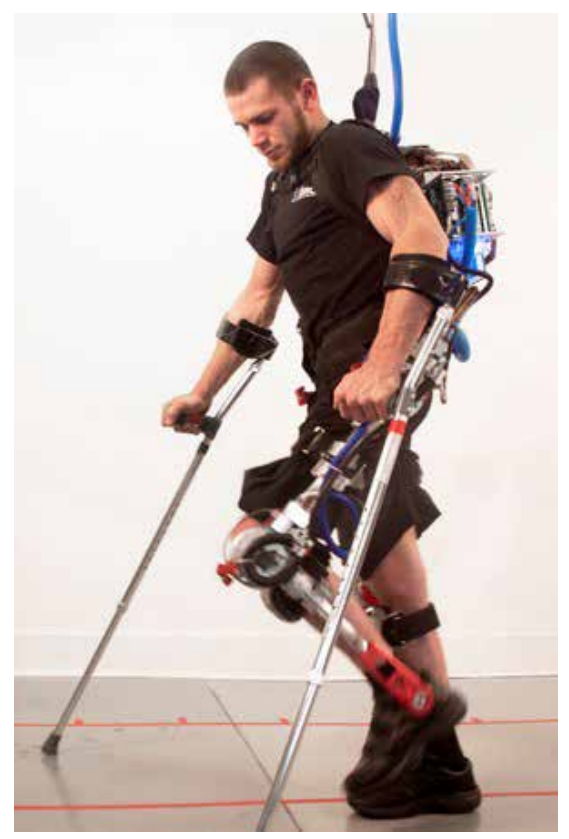

Figure 32

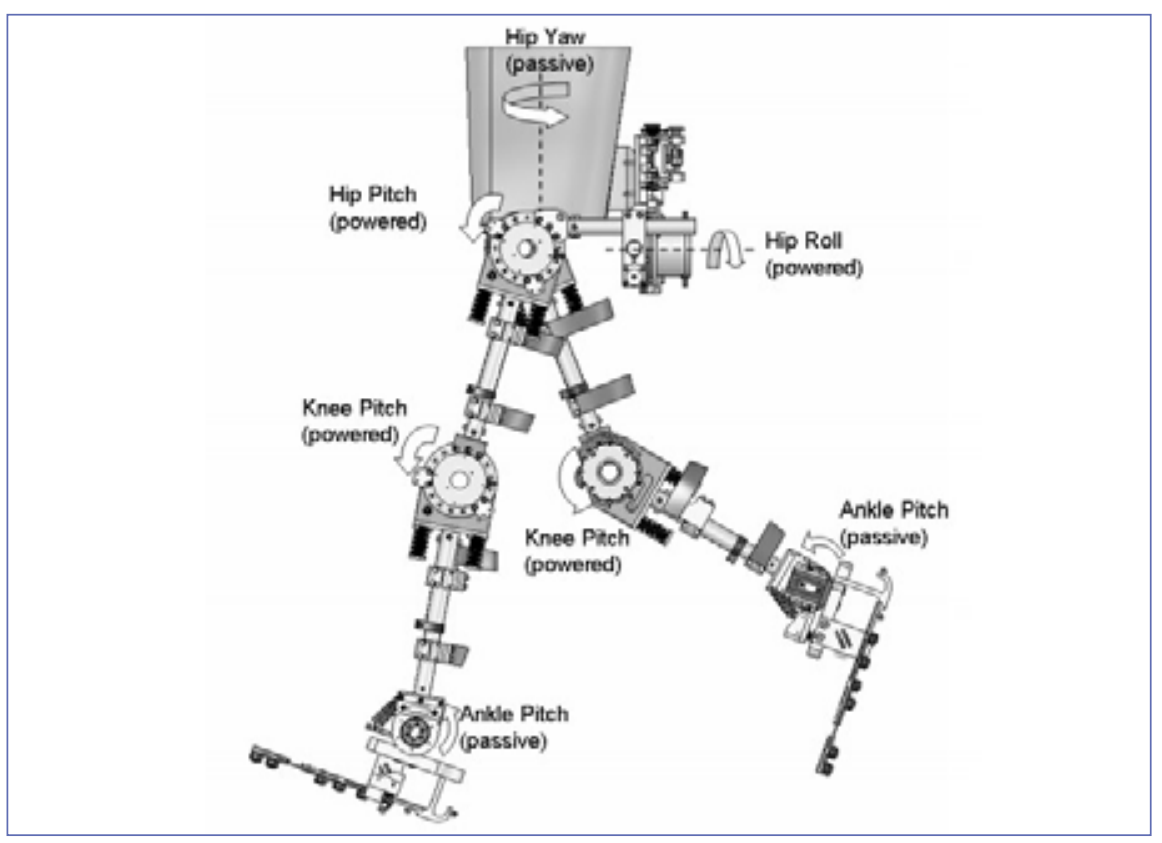

Figure 33 
attached, and the transmission of undesirable forces or torques (e.g. forces and torques parallel to the limb) at the attachment points, causing muscle torsion or attachments sliding. However, it is important to remark that the main cause is probably linked to the complexity of the human joints motions. Indeed, rotations of the joints are strongly coupled with the translation of the rotation axes.

"Unfortunately, many of the existing rebabilitation robots neglects this coupling and model human joints as a collection of simple hinges with pure rotary movements" (95).

In particular, many robots model the knee as a perfect hinge. However, in reality the knee is a complex joint that allows the femur and tibia to rotate, twist, and slide relatively to one another. Indeed, the tibiofemoral joint has six degrees of freedom: three rotations and three translations. Of course, most of the DOF of the tibiofemoral joint are mostly constrained with bony and ligamentous structures, but it is worth to be aware of what it means to neglect some of them. The literature agrees on at least four movements that cannot be neglected. The primary motion is "flexion/extension". This rotation is always coupled to a tibiofemoral anterior-posterior translation whose magnitude can exceed $19 \mathrm{~mm}$. The rotation is also coupled to a proximal-distal translation. The coupling is due to the menisci's curvature at the contact area between the femur and tibia. The last significant motion of the human knee joint is the internal-external rotation, with a range of $50^{\circ}$ when the knee is fully flexed (98).

To solve this issue, several research groups proposed different solutions.

In 2011, L.E. Amigo et al. (94) developed a virtual joint model based on three active DOFs whose purpose is to

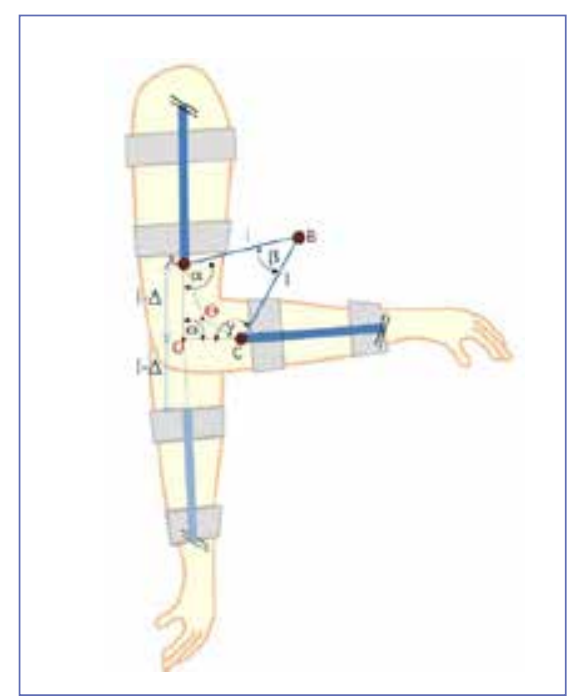

Figure 34 emulate a human joint, in particular, the elbow and the knee joints. The system is composed of a four-links chain whose two extreme links are connected to the human limbs (see figure 34). Three actuators connect the four links together at each pivot joint. To avoid the misalignment of upper/ lower limbs joints, the three actuators are driven in such a way that the exoskeleton model accommodate to different anthropometric arm/leg measures and reproduce the true movement of the joint, by avoiding the initial offset between orthosis and patient at the beginning of a therapy and compensating the migration of the Instantaneous Center of Rotation (ICR) produced by the angular joint movement. The control laws are based on a geometry study of the device that links the actuators motion with the position of the ICR coupled to the desired flexion/extension angle of the limb. Some constrains are also taken into account in the equations of motion to ensure the alignment of the mechanism with the ICR of a human joint. The model has been tested in simulation and was able to follow three different ICR pathways whose shape is typically the one of respectively a single pivot joint, an elbow joint and a knee joint. Unfortunately, when it comes to practice, the main issue of this proposal is the difficulty to know the desired ICR path that must be followed. Therefore, to know the position of the ICR and avoid the total misalignment the developers foresee the study of a model based on the measurement of interaction forces between the orthosis and the joint.

The same year (2011) and with the same purpose, i.e. to follow the instantaneous position of the knee joint rotational axis, Ergin et al. (95) developed their own robotic solution based on a 3-RRP planar parallel mechanism (figure 37). The basic unit of the 3-RRP planar mechanism is an RRP mechanism (see figure 35), where $\mathrm{R}$ stands for Revolute joint and $\mathrm{P}$ for Prismatic joint. As the name suggests an RRP mechanism consists of four links successively linked in series by three joints: two revolute joints and one prismatic joint. Of these joints, only the first rotational joint is actuated while the other two are passive. As schematized in figure 36, three RRP are put in parallel with one extreme of each RRP on the same origin point of a fixed frame (O) and the end effector linked to the same body (E). In this way the points $\mathrm{P}, \mathrm{Q}$, and $\mathrm{R}$ move along the same circle, and through them it is possible to control the 3 planar DOF of the body E. The mechanical design to obtain this kinematics is reported in figure 37 where the femur and the tibia are respectively attached to bodies $\mathrm{N}$ and $\mathrm{E}$ and where the points $\mathrm{P}, \mathrm{Q}$, and $\mathrm{R}$ moves along three identical concentric circles, respectively $\mathrm{S}, \mathrm{T}$, and $\mathrm{V}$, each actuated by a graphite brushed DC motors. Optical encoders are attached to the motors. The developers based the controller of the device on a one to one correspondence between rotation and trans- 


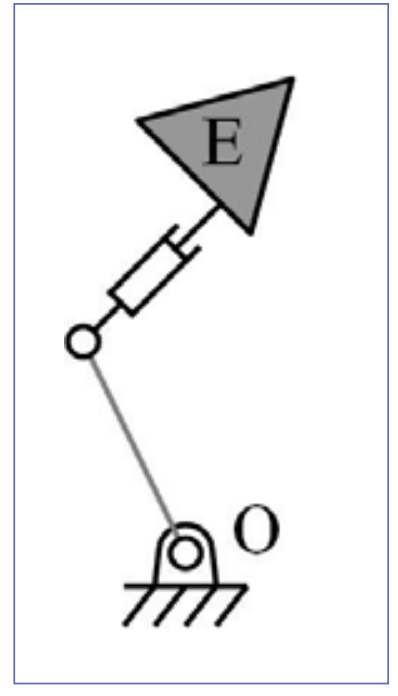

Figure 35

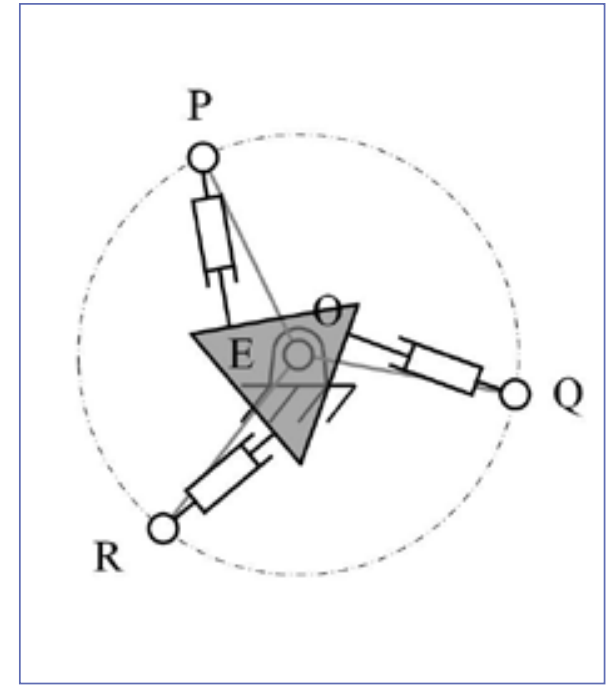

Figure 36

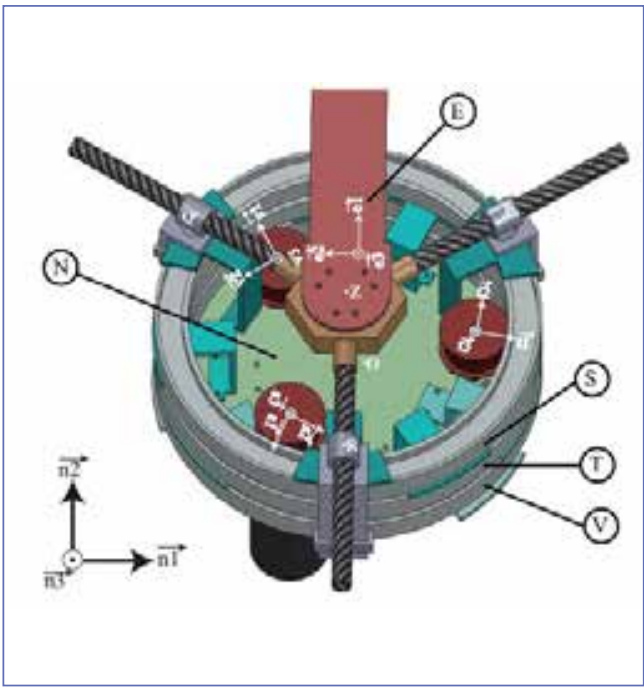

Figure 37

lation of the tibiofemoral joint, for example, a $60^{\circ}$ rotation of the device is commanded simultaneously with a $15 \mathrm{~mm}$ translation of the rotation axis. Note that while the machine is able to move the center of rotation of the robotic device, there is no guarantee that this will be aligned with the center of rotation of the knee as the device is unable to measure the position of the instantaneous center of the knee. Since the current prototype is implemented only as a proof of concept, the mechanism dimensions are not optimized to minimize the footprint of the robot on the patient. Therefore, as part of the future work, the developers foresee to minimize the size of the second prototype, use higher transmission ratio to increase the torques, and test usability with health volunteers.

In 2011, Cai et al. (96) proposed a self-adjusting orthosis device for the human knee joint (see figure 38), able to compensate misalignment with the tibiofemoral joint. The main feature of this exoskeleton is that, unlike the other devices before it, it can move the knee in flexion/extension while leaving completely free the remaining 5 DOFs of the tibiofemoral joint, i.e. without constraining them. This was made possible by aligning the rotation axis of the device with the articulation of the subject. The two extremities of the device are respectively linked to thigh and shin of the user. The alignment is ensured by an original RRRPRR mechanism consisting of a serial combination of three revolute joints, one prismatic joint and two revolute joints. Only one of the six DOFs is actuated and drives the knee flexion-extension by transmitting a torque up to $40 \mathrm{Nm}$ between the thigh and the shin through the use of a brushless motor and a two-stages, backdrivable, 100:1 transmission. Each joint is equipped with a precision potentiometer whose measurements allow to compute the position of the knee helical axes that can be seen as an equivalent representation of the instantaneous rotational center of the knee. A forcetorque sensor is inserted in the load path at the extremity of the mechanical chain for the control of force. Some links can be manually adjusted to optimize performance and ensure that the mechanical chain remains

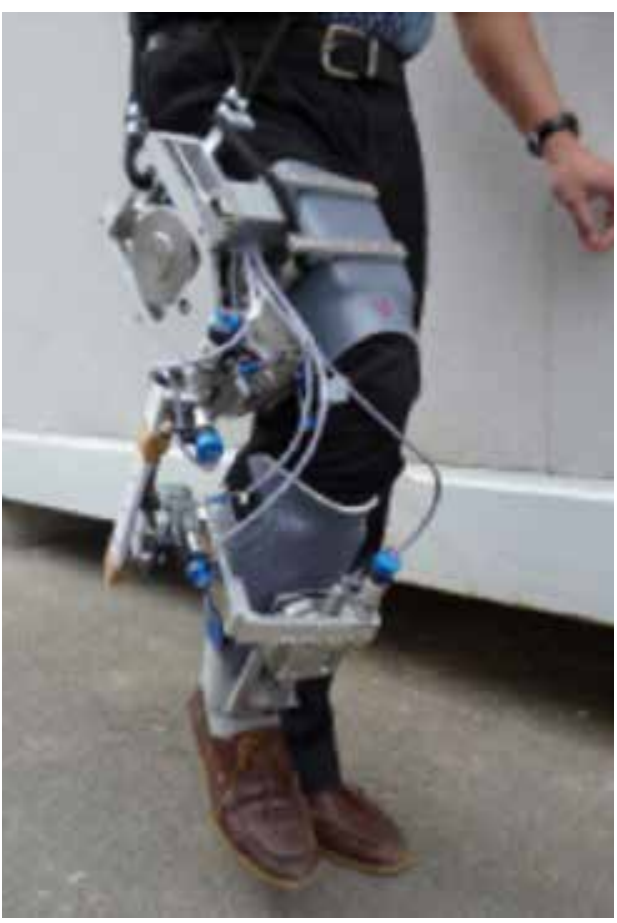

Figure 38

Muscles, Ligaments and Tendons Journal 2019;9 (1) 
far from its singularities. Currently, the size of the device is one of its main inconvenient. Another inconvenient is that although the exoskeleton is able to align on the instantaneous center of rotation of a healthy patient's knee, this ability does not help with a pathological knee. In this case, the exoskeleton is indeed not able to push the pathological instantaneous helical axes of the patient to where it might be in healthy conditions. To solve that issue and to avoid injuries, the developers used the machine in combination with a medical orthosis that guides the patient's knee following a defined physiological motion. This solution unfortunately leads to a system that globally constrains the knee since the device aligns on the instantaneous axes of the orthosis that constrains the knee. Although this solution was taken on, the developers performed experimental tests to show the accuracy and the repeatability of the measurements of the helical axes and conclude the machine is able to align on the instantaneous helical axes of the knee while performing flexion/extension.

In 2013 Celebi et al. developed ASSISTON-KNEE (97), a robotic solution that provides assistance during the flexion/ extension of the knee joint, while simultaneously accommodating and measuring the anterior-posterior translation. ASSISTON-KNEE (see figure 39) consists of a knee brace used to attach the exoskeleton to the thigh and shank of the patient, while thigh and shank links are connected to each other through a Schmidt Coupling on the lateral side, and an unactuated RRR serial mechanism on the medial side of the knee. The Schmidt Coupling is actuated by a series elastic actuator driven by Bowden cables. The main asset of Bowden cable drive is that the actuator can be remotely located, resulting in a lightweight design with low apparent inertia. The part of the exoskeleton that is connected to human limbs weighs less than $1.4 \mathrm{~kg}$. However, this asset can turn into a weakness for an exoskeleton, i.e. a wearable robot, since a remote actuation implies a fixed-based structure. The remotely located actuation unit of the Bowden cables consists of a 200W graphite brushed DC motor instrumented with an optical incremental encoder. Incremental encoders are also attached to the Schmidt coupling to measure the deflection between the mechanism disks that makes easier the forward kinematics calculation of the input torque. ASSISTON-KNEE design alleviates the need for high-precision force sensors/actuators/power transmission elements and allows for precise control of the force exerted by Bowden cable-driven actuator through typical position control of the deflection of the compliant coupling element. High-precision actuators/power transmission elements are not needed since high gain/robust position controllers can be implemented at very fast loop rates.

The developers performed feasibility tests on healthy volunteers to assess the ability of ASSISTON-KNEE to assist knee movements. Firstly, rotational flexion/extension movement were imposed to the subject, while AP translations in the sagittal plane were measured. Secondly, torque tracking performance of ASSISTON-KNEE was tested under

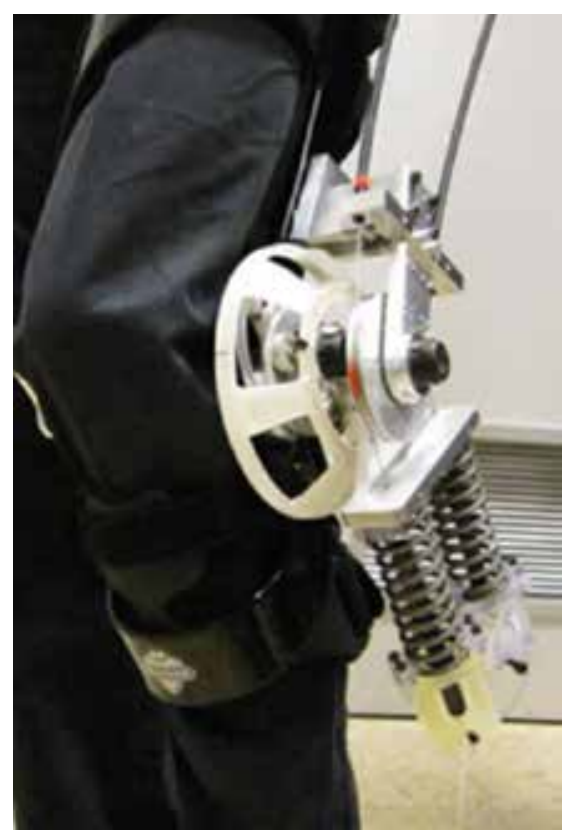

Figure 39

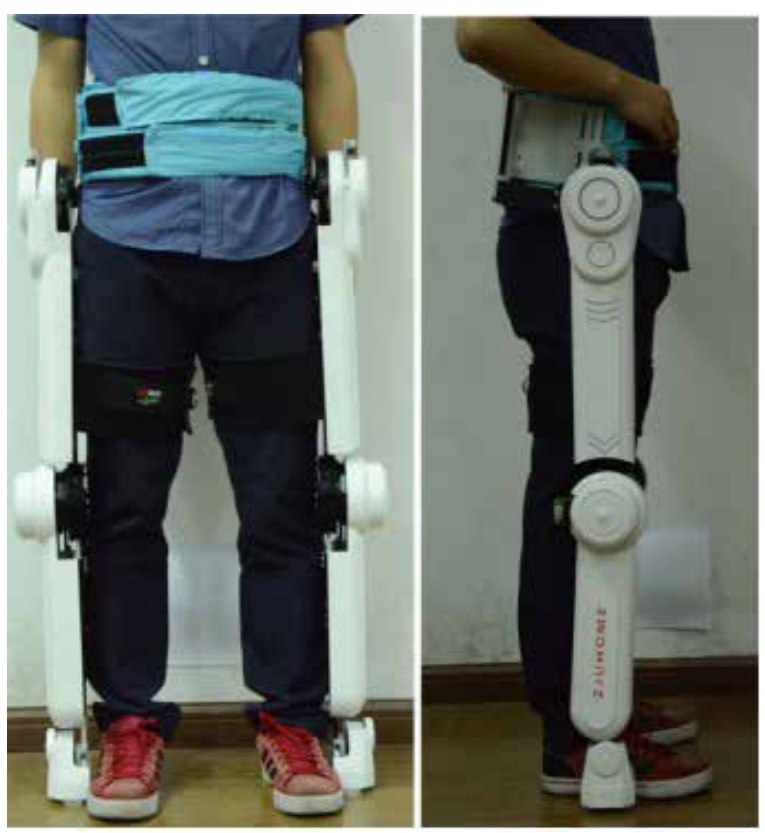

Figure 40 
explicit force control. The tests allowed to conclude that ASSISTON-KNEE is able to measure AP translations in the sagittal plane. However, the developers foresee larger scale human subject experiments and tracking of human gait with/ without the device to verify that the devices does not interfere with natural walking gait of its users. The reader might note that, unlike Cai et al.'s device, ASSISTON-KNEE does not allow the internal/external rotation of the human knee. To justify their choice, Celebi et al. argued that internal/ external rotation is severely constrained when it is loaded under body weight or fully extended (92). However, when it comes to rehabilitation, the knee is seldom fully extended or under $100 \%$ body weight.

The same year (2013), Wang et al. (99) analyzed different types of configurations for an adaptive knee exoskeleton structure and concluded that the use of a cam profile mimicking the knee kinematics would be the best option if feasible.

In 2015, a new prototype of exoskeleton (see figure 40) using bionic design of joints was presented in (100) with a new method to design lower body exoskeleton based on optimizing the human-exoskeleton physical interface to improve user comfort.

In 2015, after developing the self-adjusting isostatic exoskeleton (see figure 38), Cai et al. (101) based on the same principle to design a knee-assistive device (see figure 41) with the difference that the study focuses on gait phase detection

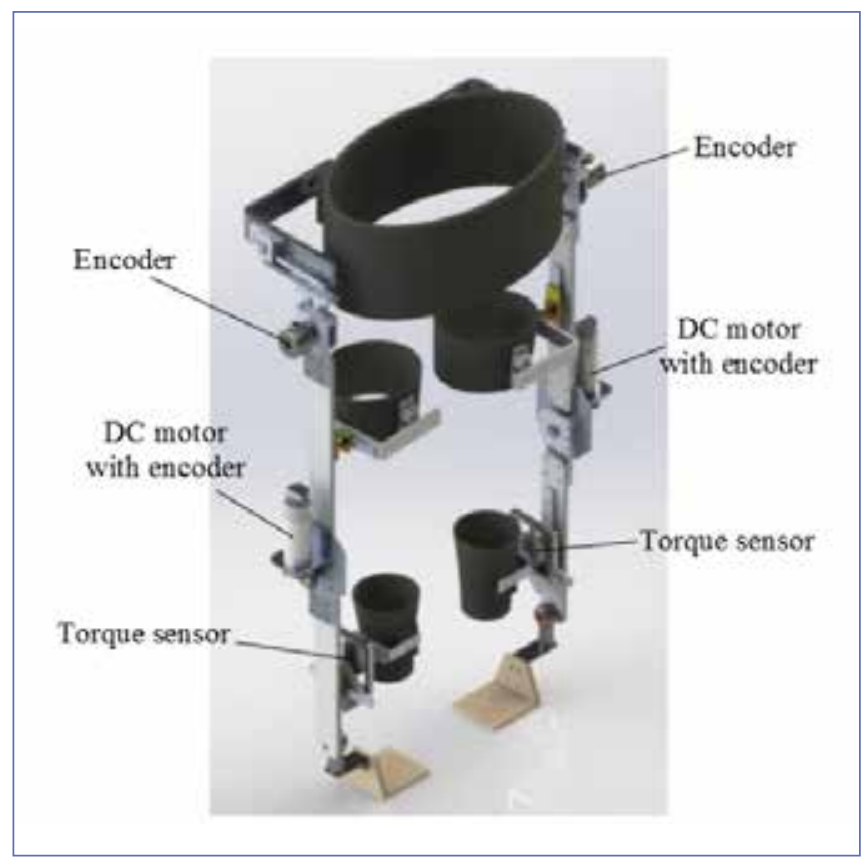

Figure 41 based predictive control. The device consists of an external structure and four internal linkages located at the hip, knee and ankle joints connecting the external structure to the user's limbs. The external structure is composed by one spherical joint located at each ankle, one motorized rotational joint located at each knee, two intersecting rotational joints at each hip and two prismatic joints which connect the whole structure to the user's back. Unlike the previous device built in 2011 by the same developers, there is only one encoder at each knee and at each side of the hip. As a consequence, although the design of the current knee-assistive device allows the natural motion of the subject knee by leaving unconstrained the 6 DOFs of the tibiofemoral joint, the actual device is not able to measure the position of the instantaneous helical axes of the knee. However, this is not an issue for an assistive device. Indeed its main purpose is not to cure a defective motion due to an instable knee, but to assist the knee and provide enough power to help the patient in achieving a goal. In 2017, the developers enhanced the transparency of the device by integrating in the motor torques computation the compensation of the inertial and gravitational effects (102).

In 2013, with the goal of reducing the size and weight of the exoskeletons, Wehner et al. (103) considered that rigid structure of the exoskeletons could influence the kinematic of the gait because of added weight or misalignments between humans' and robots' joints. With this in mind, they designed a soft lower-extremity robotic exosuit (see figure 42) seeking to augment normal muscle function in healthy individuals. They substituted the rigid beams of the exoskeletons by a suit made of resistant textile, on which pneumatic actuators are scattered in a kind of network to simulate the agonist/antagonist behavior of the muscular contraction i.e. by applying only traction. Compared to previous

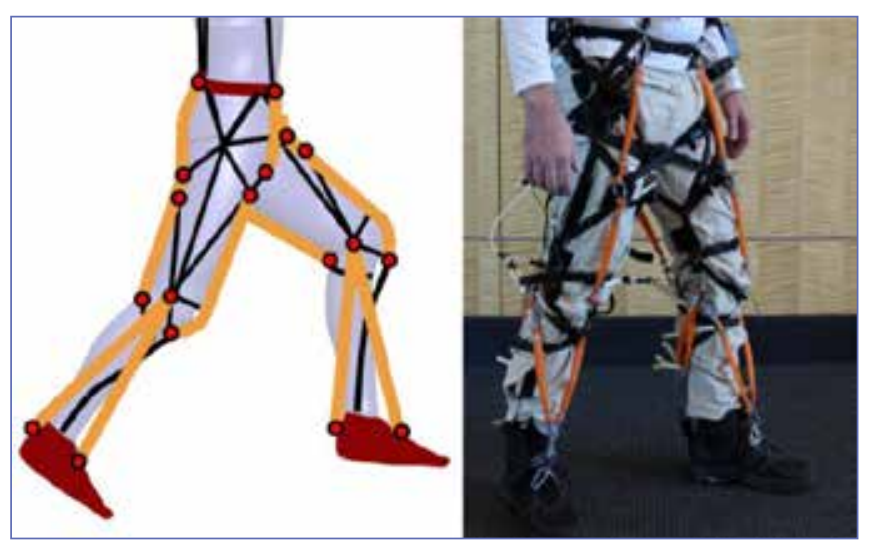

Figure 42 
exoskeletons, the device is ultra-lightweight, resulting in low mechanical impedance and inertia. Although its original purpose is power amplification, robotic exosuits could be of great interest in the future of rehabilitation robotics. However, at the current stage, although this design seems to not constrain the knee joint, it is unclear if it can help to change the center of rotation of the knee.

\section{DISCUSSION}

The effectiveness of robotic rehabilitation for motor recovery and for treatment of neurological injuries is a fact proved by many clinical trials and has a huge potential to enhance the locomotive autonomy of the patient. Over the last decades, most of the research focused on enabling active involvement of the patients through the rehabilitation process. A common solution is the progressive evolution toward the design of compliant robots whose control algorithms assist the patient only as much as needed while maintaining safe the human-robot interaction (97). This evolution from purely passive devices to compliant robots promoting active involvement of patients, has significantly increased the effectiveness of robot assisted therapies (95).

Although it is generally agreed that robotics may benefit to physiotherapy due to its ability to repeat tasks with accuracy and simultaneously measure the progress of the rehabilitation, the current knowledge does not allow researchers to define a set of guidelines that dictate the optimal design of robotic rehabilitation devices. Nevertheless, some rules might be useful to choose among different types of robots which type is the most relevant according to its application. First, it is important to remark that the choice between end-effector structures and wearable robots has major implications on the design of the rehabilitation system. Indeed, the end-effector approach involves the use of robotic arms to guide parts of the patient's anatomy along predefined trajectories. The interaction between the driven member and the robotic arm often occurs near the extremity of the respective kinematic chains. This approach is usually easier to implement but can lead to a lack of efficiency and security (96). Indeed, as the device engage several joints simultaneously, an unconstrained articulation might be moved in arbitrary directions and cause a non-physiological movement, e.g. hyper-extension. Moreover, such devices do not allow to exercise joints individually. As a consequence, diagnosis, treatment monitoring and joint-specific protocols are more difficult to introduce. Unlike the end-effector structures, the wearable robots approach is based on the individual mobility of each joint. Wearable robots usually coordinate multiple contacts and are mostly represented by exoskeleton mechanical structures (96). To choose between fixed-based end-effector systems and wearable robots, and besides the trivial choice based on ambulatory and portability issues, Krebs et al. (104) uses a kinematics criterion depending on the application field and, in particular, on the range of motion given to the patient by the machine: End-effector systems are recommended when limb segment movements require less than 45 degrees, while exoskeletons are more convenient for larger angles. Whenever a portable solution is expected, exoskeletal structures are likely more convenient (7).

Second, it is important that the robot architecture and kinematics respect the human anatomy and physiology. Indeed, both end-effector and exoskeletal systems have a direct interaction with the human user. In particular, the exoskeletons map onto several human anatomical joints and potential kinematic incompatibilities can lead to undesired interactions and/or movements with the constrained limbs. One incompatibility has already been identified on several machines, i.e. the assumption that the knee joint can be reduced to a simple hinge. This assumption leads to design rehabilitation devices that prevent the anterior-posterior translation of the tibiofemoral joint during flexion extension and constrain the rotation of the femur about the longitudinal tibial axis. Another incompatibility comes from the physical interaction between the patient and the machine: As the rigid parts of the robot are attached to human soft-tissues, the elasticity of the human-machine connection leads to joint misalignments (7). Although this issue has been particularly identified in the context of exoskeletons design, it is not limited to this unique category of machines. Indeed, at the authors' knowledge, no MTT machines nor gait trainers have been design without considering the kinematics of human knee as a pure rotary motion. For instance, the most popular lower-limb rehabilitation robot, Lokomat, employs a DC motor actuating a simple revolute joint at its knee joint (95). It is crucial to solve this problem because axis misalignment promotes compensatory movements that can inhibit recovery and decrease real life use of the limb.

Finally, unlike industrial robots that traditionally execute movements between two known points along a defined trajectory with a focus on maintaining a highly accurate position, rehabilitation robots are usually expected to be programmable and adjustable (10). Of course, the programming must rely on the rehabilitation process that aims to sequentially restore i) the flexibility and range of motion of the knee, ii) the strength and muscle endurance, and finally iii) the proprioception, coordination and agility. Thus, the proportion of passive exercises is commonly higher at the beginning and gradually decreased to move on to active exercises. Consequently, if the rehabilitation robot is meant to be used for the entire rehabilitation duration, its ability 
to perform both passive and active exercises is very important. On the contrary, it can be worth to consider the use of different specific machines focusing on different stage of the rehabilitation process. Moreover, it is commonly accepted that task specificity, selectivity and active participation by a subject are essential to determine the success of the training (i.e., faster and better recovery). Besides these points, there is no general consensus in orthopedic literature about the superiority of specific training approaches.

However, another issue might hinder the development of effective rehabilitation robots, i.e. the lack of clinical relevance allowing to assess the utility and effectiveness of robotic therapy, in particular for knee rehabilitation. This is probably due to the fact that, until now, most of the studies conducted in that field have remained at the research level and almost never completed a further step into clinical studies. Another explanation might also be that, at the current stage, there is no study assessing the benefits of the rehabilitation versus the cost due to the robotics use. Indeed, it might be noticeable to compare costs and benefits produced by the classical physiotherapy with those produced by the robots. Since robotics costs are higher than those of the classical physiotherapy, benefits produced by robotics are also expected to be higher.

It is also important to notice that when the clinical studies exist, the results of different studies are sometimes contradictory and therefore cast doubt on the utility/effectiveness of the robotic devices. This is not without reminding the controversy surrounding the CPM machines whose effectiveness for rehabilitation is, at the current, not yet clearly accepted. This issue raises to another one, i.e. the lack of reliable and quantitative methods, in both the clinical and technical literatures, to evaluate the rehabilitation progress of the patient. These methods might therefore help to clarify the utility and effectiveness of a given robotic tool regarding several pathologies. Moreover, they might also be useful to the physiotherapists' diagnosis. Incidentally, the available robots are usually designed while focusing on the Patient-Robot interaction, although the quality of this interaction is essential, but it is important to not forget that the robot takes place in between the Patient-Physiotherapist relationship (see figure 43). As a consequence, the robot must be thought as a tool used by both the patient and the practitioner. The practitioner and the patient are therefore entitled to request a different feedback for their specific use, i.e. the qualitative and quantitative information provided by the system must be relevant and specifically oriented. For instance, a "user-friendly" Graphical User Interface should allow them to learn easily from the robot, otherwise, this might be a brake on the robot distribution. At the current stage, at the best of the authors' knowledge, there is no

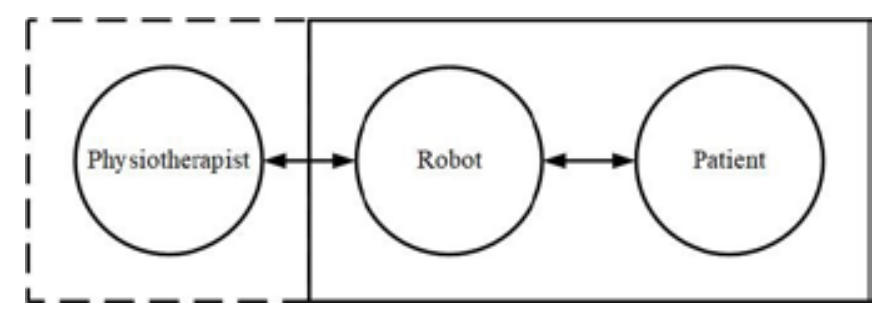

Figure 43

consensus regarding the information needed by the physiotherapist to refine his diagnosis.

\section{CONCLUSION}

In this paper, we have surveyed the state of the art of the rehabilitation robots that are currently available on the market and/or have been presented in the academic field. The robots have been classified into three categories according to their operative mode:

- Continuous Passive Motion (CPM) machines \& Therapeutic Exercise Machines (TEM);

- Gait-training Robots;

- Exoskeletons.

One of the main messages and results of this paper is that rehabilitation robotics is a multidisciplinary and multifactorial field involving a mix of competence. Indeed, besides a solid knowledge of robotics, research and development in robotic rehabilitation devices requires a deep understanding of the biomechanics of the human body and of the human neurological and cognitive systems. Indeed, bioinspired and biomimetic design are fundamental for reproducing human functionalities in a correct way (8).

Indeed, rehabilitation robots designed with insufficient knowledge of joint motions can hamper the rehabilitation process or even damage human joints. Because of this, physiological bio-joint kinematics should always be accounted when designing a rehabilitation robot. (56)

In this perspective, it is important to remark that the knee joint is a complex joint that allows the femur and tibia to rotate, twist, and slide relatively to one another. The tibiofemoral joint has six degrees of freedom - three rotations and three translations. The primary motion, of course, is flexion and extension but the other motions (and in particular the external-internal rotation and the anterior-posterior translation) are important to ensure the stability of the joint and any additional constrain to the joint motion is transformed in reaction joint force that, applied to the human structure, could produce mechanical damage. 
Unfortunately, at the current stage, a very limited number of devices takes (and often only partially) this anatomical fact into account, while most of the machines only focus on the primary flexion and extension motion and neglect the others. Another message of this paper is that, although several robotic rehabilitation systems for the lower limbs have been proposed, there is a quite limited number of clinical trials with clear goals and solid methodology that demonstrate in a definitive way the effect of the use of robotic devices in the rehabilitation process. A consequence of this lack of systematic clinical trials is that, it is still unclear what characteristics should be incorporated in a rehabilitation robot to enhance its effectiveness (10) and a clear comparison among system it doesn't exist.

In the future, robotic therapy might have real benefits complementing clinical practice, e.g. by reducing therapists' workload and work-related pathologies, by decreasing cost and time involved in the rehabilitation process, by providing more extensive therapeutic programs, and by providing quantitative measures to evaluate the patients' progress along the rehabilitation process (10). Indeed, unlike the scoring approach currently used as a rehabilitation assessment tool by physiotherapists, haptic and robotic technologies might be a key asset to provide objective measurement during exercise and be the next stage in rehabilitation.

\section{REFERENCES}

1. OECD, OCDE, EU, UE. Health at a Glance: Europe 2016: State of Health in the EU Cycle. OECD Publishing; Éditions OCDE; 2016.

2. Csintalan RP, Inacio MCS, Funahashi TT. Incidence Rate of Anterior Cruciate Ligament Reconstructions. Perm J. 2008;12(3):17-21.

3. Bracht H, Goubau L, Stuyts B, Schepens A, Verdonk P, Victor J. Surgical management of anterior cruciate ligament injuries in Belgium anno 2013. Acta orthopaedica Belgica. 2015;81:738-46.

4. Imhoff AB, Beitzel K, Stamer K, Klein E, Mazzocca G, editors. Rehabilitation in Orthopedic Surgery. Berlin, Heidelberg: Springer Berlin Heidelberg; 2016.

5. Glover W. Work-related Strain Injuries in Physiotherapists. Physiotherapy. 2002 Jun;88(6):364-72.

6. Salter RB, Hamilton HW, Wedge JH, Tile M, Torode IP, O'Driscoll SW, et al. Clinical application of basic research on continuous passive motion for disorders and injuries of synovial joints: A preliminary report of a feasibility study. Journal of Orthopaedic Research. 1983;1(3):325-42.

7. Rocon E, Pons JL. Exoskeletons in rehabilitation robotics: tremor suppression. Berlin: Springer; 2011. 138 p. (Springer tracts in advanced robotics).

8. Siciliano B, Khatib O, editors. Springer handbook of robotics. Berlin: Springer; 2008. 1611 p.
9. Rimec S.r.l.: CPM lower limbs [Internet]. [cited $2017 \mathrm{Nov}$ 21]. Available from: http://fisiotek3000.blogspot.com/p/ cpm-lower-limbs_18.html

10. Marko M, Tadej B. Rehabilitation robotics. Technology and Health Care. 2011;(6):483-495.

11. Pons JL, editor. Wearable robots: biomechatronic exoskeletons. Hoboken, N.J: Wiley; 2008. 338 p.

12. GE's Retro exoskeleton (robot) from the 1950s « Adafruit Industries - Makers, hackers, artists, designers and engineers! [Internet]. [cited 2017 Nov 21]. Available from: https:// blog.adafruit.com/2010/09/08/ges-retro-exoskeleton-robot-from-the-1950s/

13. Robert Salter [Internet]. [cited 2017 Nov 21]. Available from: http://continuouspassivemotion.org/Pages/rbsalter.htm

14. Salter RB, Hamilton HW, Wedge JH, Tile M, Torode IP, O'Driscoll SW, et al. Clinical application of basic research on continuous passive motion for disorders and injuries of synovial joints: A preliminary report of a feasibility study. Journal of Orthopaedic Research. 1983;1(3):325-42.

15. Smith TO, Davies L. The efficacy of continuous passive motion after anterior cruciate ligament reconstruction: A systematic review. Physical Therapy in Sport. 2007 Aug;8(3):141-52.

16. Bennett LA, Brearley SC, Hart JAL, Bailey MJ. A comparison of 2 continuous passive motion protocols after total knee arthroplasty: A controlled and randomized study. Journal of Arthroplasty. 2005;20(2):225 - 233.

17. Maniar RN, Baviskar JV, Singhi T, Rathi SS. To Use or Not to Use Continuous Passive Motion Post-Total Knee Arthroplasty. The Journal of Arthroplasty. 2012 Feb;27(2):193-200.e1.

18. Akdoğan E, Adli MA. The design and control of a therapeutic exercise robot for lower limb rehabilitation: Physiotherabot. Mechatronics. 2011 Apr;21(3):509-22.

19. Witherow GE, Bollen SR, Pinczewski LA. The use of continuous passive motion after arthroscopically assisted anterior cruciate ligament reconstruction: help or hindrance? Knee Surgery, Sports Traumatology, Arthroscopy. 1993 Jun;1(2):68-70.

20. Denis M, Moffet H, Caron F, Ouellet D, Paquet J, Nolet L. Effectiveness of continuous passive motion and conventional physical therapy after total knee arthroplasty: a randomized clinical trial. Phys Ther. 2006 Feb;86(2):174-85.

21. Boese CK, Weis M, Phillips T, Lawton-Peters S, Gallo T, Centeno L. The Efficacy of Continuous Passive Motion After Total Knee Arthroplasty: A Comparison of Three Protocols. The Journal of Arthroplasty. 2014 Jun;29(6):1158-62.

22. Krukowski R. Particle brake clutch muscle exercise and rehabilitation apparatus. 4765315, 1988. p. 12.

23. Khalili D, Zomlefer M. An intelligent robotic system for rehabilitation of joints and estimation of body segment parameters. IEEE Transactions on Biomedical Engineering. 1988 Feb;35(2):138-46.

24. Dijkers MP, deBear PC, Erlandson RF, Kristy K, Geer DM, Nichols A. Patient and staff acceptance of robotic technology in occupational therapy: A pilot study. The Journal of Rehabilitation Research and Development. 1991;28(2):33.

25. Biodex [Internet]. [cited 2017 Nov 21]. Available from: http://www.cmslaval.com/biodex.html

26. Moughamir S, Manamanni N, Zaytoon J, Afilal L. Control law implementation for Multi-Iso: a training machine for 
lower limbs. In: 2001 Conference Proceedings of the 23rd Annual International Conference of the IEEE Engineering in Medicine and Biology Society [Internet]. Istanbul, Turkey: IEEE; 2001 [cited 2019 Feb 16]. p. 1477-80. Available from: http://ieeexplore.ieee.org/document/1020484/

27. Bradley D, Acosta-Marquez C, Hawley M, Brownsell S, Enderby P, Mawson S. NeXOS - The design, development and evaluation of a rehabilitation system for the lower limbs. Mechatronics. 2009 Mar;19(2):247-57.

28. Hu W, Li G, Sun Y, Jiang G, Kong J, Ju Z, et al. A Review of Upper and Lower Limb Rehabilitation Training Robot. In: Huang Y, Wu H, Liu H, Yin Z, editors. Intelligent Robotics and Applications. Cham: Springer International Publishing; 2017. p. $570-80$

29. Physiotherabot (Physiotherapist Robot) [Internet]. [cited 2017 Nov 21]. Available from: http://erhanakdogan. com/?page_id=2237

30. Reha-Stim [Internet]. [cited 2017 Nov 21]. Available from: http://www.reha-stim.de/cms/index.php?id=76

31. Jezernik S, Colombo G, Keller T, Frueh H, Morari M. Robotic Orthosis Lokomat: A Rehabilitation and Research Tool: Robotic Orthosis Lokomat. Neuromodulation: Technology at the Neural Interface. 2003 Apr;6(2):108-15.

32. Hospital Newspaper [Internet]. [cited 2017 Nov 21]. Available from: http://www.hospitalnewspaper.com/webpages/news/displaynews.aspx?PT $=$ features $\&$ cat $=$ The $\% 20$ Cutting \%20edge \&ID=c767f17d-5499-4060-b77e-7f51afa30cbe

33. Hesse $\mathrm{S}$, Uhlenbrock $\mathrm{D}$. A mechanized gait trainer for restoration of gait. J Rehabil Res Dev. 2000 Dec;37(6):701-8.

34. Werner C, Pohl M, Holzgraefe M, Kroczek G, Mehrholz J, Wingendorf I, et al. Lokomotionstherapie des akuten Schlaganfallpatienten: Ergebnisse der multizentrischen Deutschen Gangtrainer Studie (DEGAS). Neurologie \& Rehabilitation. 2006;12(5):262-9.

35. Tong RK, Ng MF, Li LS. Effectiveness of Gait Training Using an Electromechanical Gait Trainer, With and Without Functional Electric Stimulation, in Subacute Stroke: A Randomized Controlled Trial. Archives of Physical Medicine and Rehabilitation. 2006 Oct;87(10):1298-304.

36. Reha-Stim [Internet]. [cited 2017 Nov 21]. Available from: https://reha-stim.com/cms/?id=94

37. Reha-Stim [Internet]. [cited 2017 Nov 21]. Available from: https://reha-stim.com/cms/assets/files/Flyer/Gait\%20Trainer\%20GT\%20I\%20Flyer\%20Englisch\%202012.pdf

38. Legal notes - Hocoma [Internet]. [cited 2017 Nov 21]. Available from: https:/www.hocoma.com/legal-notes/\#lokomatpro_lokomatnanos

39. Lünenburger L, Colombo G, Riener R, Dietz V. Clinical Assessments Performed During Robotic Rehabilitation by the Gait Training Robot Lokomat. In: 9th International Conference on Rehabilitation Robotics, 2005 ICORR 2005. Chicago, IL, USA: IEEE; 2005. p. 345-8.

40. Hidler J, Nichols D, Pelliccio M, Brady K, Campbell DD, Kahn JH, et al. Multicenter Randomized Clinical Trial Evaluating the Effectiveness of the Lokomat in Subacute Stroke. Neurorehabilitation and Neural Repair. 2009 Jan;23(1):5-13.

41. Westlake KP, Patten C. Pilot study of Lokomat versus manual-assisted treadmill training for locomotor recovery post- stroke. Journal of NeuroEngineering and Rehabilitation. 2009 Dec;6(1).

42. Chung BPH. Effectiveness of robotic-assisted gait training in stroke rehabilitation: A retrospective matched control study. Hong Kong Physiotherapy Journal. 2017 Jun;36:10-6.

43. Ichinose WE, Reinkensmeyer DJ, Aoyagi D, Lin JT, Ngai $\mathrm{K}$, Edgerton VR, et al. A robotic device for measuring and controlling pelvic motion during locomotor rehabilitation. In: Proceedings of the 25th Annual International Conference of the IEEE Engineering in Medicine and Biology Society (IEEE Cat No03CH37439). Cancun, Mexico: IEEE; 2003. p. 1690-3.

44. Aoyagi D. A Robotic Device for Human Gait Rehabilitation: Achieving naturalistic assistance for the pelvis and legs through compliant pneumatic actuation and temporal synchronization [PhD Thesis]. University of California, Irvine; 2006.

45. Reinkensmeyer DJ, Aoyagi D, Emken JL, Galvez JA, Ichinose W, Kerdanyan G, et al. Tools for understanding and optimizing robotic gait training. J Rehabil Res Dev. 2006 Sep;43(5):657-70.

46. Schmidt H. HapticWalker - A novel haptic device for walking simulation. In: Proceedings of EuroHaptics Conference 2004. Munich, Germany; 2004. p. 60-7.

47. Hussein S, Schmidt H, Volkmar M, Werner C, Helmich I, Piorko F, et al. Muscle coordination in healthy subjects during floor walking and stair climbing in robot assisted gait training. In: 2008 30th Annual International Conference of the IEEE Engineering in Medicine and Biology Society. Vancouver, BC: IEEE; 2008. p. 1961-4.

48. Sale P, Franceschini M, Waldner A, Hesse S. Use of the robot assisted gait therapy in rehabilitation of patients with stroke and spinal cord injury. Eur J Phys Rehabil Med. 2012 Mar;48(1):111-21.

49. Veneman JF, Ekkelenkamp R, Kruidhof R, van der Helm FCT, van der Kooij H. Design of a Series Elastic and Bowdencable-Based Actuation System for Use As Torque-Actuator in Exoskeleton-Type Training Robots. In: 9th International Conference on Rehabilitation Robotics, 2005 ICORR 2005. Chicago, IL, USA: IEEE; 2005. p. 496-9.

50. Meuleman J, van Asseldonk EHF, van der Kooij H. Novel actuation design of a gait trainer with shadow leg approach. In: 2013 IEEE 13th International Conference on Rehabilitation Robotics (ICORR). Seattle, WA: IEEE; 2013. p. 1-8.

51. Meuleman J, van Asseldonk E, van Oort G, Rietman H, van der Kooij H. LOPES II-Design and Evaluation of an Admittance Controlled Gait Training Robot With Shadow-Leg Approach. IEEE Transactions on Neural Systems and Rehabilitation Engineering. 2016 Mar;24(3):352-63.

52. Banala SK, Agrawal SK, Scholz JP. Active Leg Exoskeleton (ALEX) for Gait Rehabilitation of Motor-Impaired Patients. In: 2007 IEEE 10th International Conference on Rehabilitation Robotics. Noordwijk, Netherlands: IEEE; 2007. p. 401-7.

53. Rutherford DJ, Hubley-Kozey C. Explaining the hip adduction moment variability during gait: Implications for hip abductor strengthening. Clinical Biomechanics. 2009 Mar;24(3):267-73.

54. Reinkensmeyer DJ, Aoyagi D, Emken JL, Galvez JA, Ichinose W, Kerdanyan G, et al. Tools for understanding and 
optimizing robotic gait training. J Rehabil Res Dev. 2006 Sep;43(5):657-70.

55. Zanotto D, Stegall P, Agrawal SK. ALEX III: A novel robotic platform with 12 DOFs for human gait training. In: 2013 IEEE International Conference on Robotics and Automation. Karlsruhe, Germany: IEEE; 2013. p. 3914-9.

56. Stegall P, Zanotto D, Agrawal SK. Variable Damping Force Tunnel for Gait Training Using ALEX III. IEEE Robotics and Automation Letters. 2017 Jul;2(3):1495-501.

57. Jin X, Cui X, Agrawal SK. Design of a cable-driven active leg exoskeleton (C-ALEX) and gait training experiments with human subjects. In: 2015 IEEE International Conference on Robotics and Automation (ICRA). Seattle, WA, USA: IEEE; 2015. p. 5578-83.

58. Surdilovic D, Bernhardt R. STRING-MAN: a new wire robot for gait rehabilitation. In: IEEE International Conference on Robotics and Automation, 2004 Proceedings ICRA '04 2004. New Orleans, LA, USA: IEEE; 2004. p. 2031-6.

59. Koceska N, Koceski S, Beomonte Zobel P, Durante F. Pneumatically actuated exoskeleton for gait rehabilitation. In: Proceedings of the 8th JFPS International Symposium on Fluid Power. Okinawa, Japan; 2011. p. 43-50.

60. Alton F, Baldey L, Caplan S, Morrissey MC. A kinematic comparison of overground and treadmill walking. Clin Biomech (Bristol, Avon). 1998 Sep;13(6):434-40.

61. Stolze H, Kuhtz-Buschbeck J., Mondwurf C, Boczek-Funcke A, Jöhnk K, Deuschl G, et al. Gait analysis during treadmill and overground locomotion in children and adults. Electroencephalography and Clinical Neurophysiology/Electromyography and Motor Control. 1997 Dec;105(6):490-7.

62. AlGheshyan FN. Comparison of Ground Reaction Force in Treadmill Walking and in Overground Walking. [Coral Gables, Florida]: University of Miami; 2012.

63. Steinicke F, Visell Y, Campos J, Lécuyer A, editors. Human Walking in Virtual Environments [Internet]. New York, NY: Springer New York; 2013 [cited 2019 Feb 17]. Available from: http://link.springer.com/10.1007/978-1-4419-8432-6

64. Panasonic Power Loader Light exoskeleton takes a load off your back [Internet]. [cited 2017 Apr 5]. Available from: https://newatlas.com/panasonic-power-loader-light-exoskeleton/25682/

65. Bogue R. Exoskeletons and robotic prosthetics: a review of recent developments. Industrial Robot: An International Journal. 2009 Aug 21;36(5):421-7.

66. 佐川電子 Sagawa Electronics,inc. [Internet]. [cited 2017 Nov 21]. Available from: http://www.sagawaelectronics.com/ index.html\#poweredjacket

67. HULCTM | Berkeley Robotics \& Human Engineering Laboratory [Internet]. [cited $2017 \mathrm{Nov}$ 21]. Available from: https://bleex.me.berkeley.edu/research/exoskeleton/hulc/

68. Raytheon XOS 2 Exoskeleton, Second-Generation Robotics Suit - Army Technology [Internet]. [cited 2017 Nov 21]. Available from: https://www.army-technology.com/projects/ raytheon-xos-2-exoskeleton-us/

69. Sankai Y. HAL: Hybrid Assistive Limb Based on Cybernics. In: Kaneko M, Nakamura Y, editors. Robotics Research [Internet]. Berlin, Heidelberg: Springer Berlin Heidelberg; 2010 [cited 2019 Feb 17]. p. 25-34. Available from: http:// link.springer.com/10.1007/978-3-642-14743-2_3
70. NIPPONIA [Internet]. [cited 2017 Nov 21]. Available from: https://web-japan.org/nipponia/nipponia38/en/feature/ feature02.html

71. EksoHealth | Ekso Bionics [Internet]. [cited 2017 Nov 21]. Available from: https://eksobionics.com/eksohealth/products/

72. Shiqian Wang, van Dijk W, van der Kooij H. Spring uses in exoskeleton actuation design. In: 2011 IEEE International Conference on Rehabilitation Robotics. Zurich: IEEE; 2011. p. 1-6.

73. Indego - Powering People Forward I Parker Indego [Internet]. [cited 2017 Nov 21]. Available from: http://www.indego.com/indego/en/home

74. PHOENIX Medical Exoskeleton | suitX [Internet]. [cited 2017 Nov 21]. Available from: https://www.suitx.com/phoenix-medical-exoskeleton

75. This robotic exoskeleton helps paralysed patients to walk and it's getting smarter [Internet]. [cited 2017 Nov 21]. Available from: https://www.wareable.com/wearable-tech/exoskeleton-paralysed-patients-ekso-bionics-gt-sarah-thomas

76. Harding N, Amundson K, Burns J, Angold R, Zoss A, Kazerooni $\mathrm{H}$. Exoskeleton load handling system and method of use. WO 2011/127421 Al, 2011.

77. Zoss A, Evans J, Sandler R, Harding N, Julin A, Lubin J, et al. Reconfigurable exoskeleton. WO 2014/093470 Al, 2014. p. 39.

78. Angold R, Fleming N. Exoskeleton and method of increasing the flexibility of an exoskeleton hip joint. US 2016/0229065 A1, 2016.

79. Kolakowsky-Hayner SA, Crew J, Moran S, Shah A. Safety and Feasibility of using the EksoTM Bionic Exoskeleton to Aid Ambulation after Spinal Cord Injury. Journal of Spine. 2013;

80. eLEGSTM | Berkeley Robotics \& Human Engineering Laboratory [Internet]. [cited 2017 Nov 21]. Available from: https://bleex.me.berkeley.edu/research/exoskeleton/ elegs $\% \mathrm{E} 2 \% 84 \% \mathrm{~A} 2 /$

81. Little R, Irving RA. Self contained powered exoskeleton walker for a disabled user. US 2011/0066088 A1, 2011. p. 56.

82. Beirne MA, Nedley K, Wallis S, Francisco GE. A Treatment Proposal with Lower Extremity Robotic Devices: Performance of Postural Analysis and Determination of Exercises Using the REX Robot. TIRR Memorial Hermann - Rehabilitation \& Research.

83. Barbetta DC, Lopes ACG, Chagas FNMR, Soares PT, Casaro FM, Poletto MF, et al. Predictors of musculoskeletal pain in the upper extremities of individuals with spinal cord injury. Spinal Cord. 2016 Feb;54(2):145-9.

84. ReWalk 6.0 - Home [Internet]. [cited 2017 Nov 21]. Available from: https://rewalk.com/

85. Raab K, Krakow K, Tripp F, Jung M. Effects of training with the ReWalk exoskeleton on quality of life in incomplete spinal cord injury: a single case study. Spinal Cord Series and Cases. 2016 Dec;2(1)

86. Asselin P, Knezevic S, Kornfeld S, Cirnigliaro C, Agranova-Breyter I, Bauman WA, et al. Heart rate and oxygen demand of powered exoskeleton-assisted walking in persons with paraplegia. Journal of Rehabilitation Research and Development. 2015;52(2):147-58. 
87. Yang A, Asselin P, Knezevic S, Kornfeld S, Spungen A. Assessment of In-Hospital Walking Velocity and Level of Assistance in a Powered Exoskeleton in Persons with Spinal Cord Injury. Topics in Spinal Cord Injury Rehabilitation. 2015 Mar;21(2):100-9.

88. Indego Exoskeleton After SCI - Tabular View - ClinicalTrials. gov [Internet]. [cited 2017 Nov 21]. Available from: https:// clinicaltrials.gov/ct2/show/record/NCT02793635

89. Austin I Berkeley Robotics \& Human Engineering Laboratory [Internet]. [cited 2017 Nov 21]. Available from: http://bleex. me.berkeley.edu/research/exoskeleton/medical-exoskeleton/

90. Horst RW. A bio-robotic leg orthosis for rehabilitation and mobility enhancement. In: 2009 Annual International Conference of the IEEE Engineering in Medicine and Biology Society. Minneapolis, MN: IEEE; 2009. p. 5030-3.

91. X1 Mina Exoskeleton - IHMC Robotics Lab [Internet]. [cited 2017 Nov 21]. Available from: http://robots.ihmc.us/ x1-mina-exoskeleton/

92. Hian Kai Kwa, Noorden JH, Missel M, Craig T, Pratt JE, Neuhaus PD. Development of the IHMC Mobility Assist Exoskeleton. In: 2009 IEEE International Conference on Robotics and Automation. Kobe: IEEE; 2009. p. 2556-62.

93. Neuhaus PD, Noorden JH, Craig TJ, Torres T, Kirschbaum J, Pratt JE. Design and evaluation of Mina: A robotic orthosis for paraplegics. In: 2011 IEEE International Conference on Rehabilitation Robotics. Zurich: IEEE; 2011. p. 1-8.

94. Amigo LE, Casals A, Amat J. Design of a 3-DoF joint system with dynamic servo-adaptation in orthotic applications. In: 2011 IEEE International Conference on Robotics and Automation. Shanghai, China: IEEE; 2011. p. 3700-5.

95. Ergin MA, Patoglu V. A self-adjusting knee exoskeleton for robot-assisted treatment of knee injuries. In: 2011 IEEE/RSJ International Conference on Intelligent Robots and Systems. San Francisco, CA: IEEE; 2011. p. 4917-22.

96. Cai VAD, Bidaud P, Hayward V, Gosselin F, Desailly E. Self-adjusting, isostatic exoskeleton for the human knee joint. In: 2011 Annual International Conference of the IEEE Engi- neering in Medicine and Biology Society. Boston, MA: IEEE; 2011. p. 612-8.

97. Celebi B, Yalcin M, Patoglu V. AssistOn-Knee: A self-aligning knee exoskeleton. In: 2013 IEEE/RSJ International Conference on Intelligent Robots and Systems. Tokyo: IEEE; 2013. p. 996-1002.

98. Bartel DL, Davy DT, Keaveny TM. Orthopaedic biomechanics: mechanics and design in musculoskeletal systems. Upper Saddle River, N.J: Pearson/Prentice Hall; 2006. 370 p. (Pearson Prentice Hall bioengineering).

99. Wang D, Lee K-M, Guo J, Yang C-J. Adaptive Knee Joint Exoskeleton Based on Biological Geometries. IEEE/ASME Transactions on Mechatronics. 2014 Aug;19(4):1268-78.

100. Yang W, Yang C, Wei Q, Zhu M. Reducing the Human-Exoskeleton Interaction Force Using Bionic Design of Joints. In: Yang C, Virk GS, Yang H, editors. Wearable Sensors and Robots. Singapore: Springer Singapore; 2017. p. 195-209.

101. Cai VAD, Bidaud P, Le TT, Ibanez A, Phan XT. In search of transparency for lower limb exoskeleton devices with a new mechanical design and a robust gait phases detection method. In: 2015 IEEE International Conference on Robotics and Biomimetics (ROBIO). Zhuhai: IEEE; 2015. p. 1151-6.

102. Cai VAD, Ibanez A, Granata C, Nguyen VT, Nguyen MT. Transparency enhancement for an active knee orthosis by a constraint-free mechanical design and a gait phase detection based predictive control. Meccanica. 2017 Feb;52(3):729-48.

103. Wehner M, Quinlivan B, Aubin PM, Martinez-Villalpando E, Baumann M, Stirling L, et al. A lightweight soft exosuit for gait assistance. In: 2013 IEEE International Conference on Robotics and Automation. Karlsruhe, Germany: IEEE; 2013. p. 3362-9.

104. Krebs H, Dipietro L, Levy-Tzedek S, Fasoli S, Rykman-Berland A, Zipse J, et al. A paradigm shift for rehabilitation robotics. IEEE Engineering in Medicine and Biology Magazine. 2008 Jul;27(4):61-70.

105. Krebs HI, Volpe BT. Rehabilitation Robotics. In: Handbook of Clinical Neurology. Elsevier; 2013. p. 283-94. 